\title{
Title: Epoxy fatty acid dysregulation and neuroinflammation in Alzheimer's disease is resolved by a soluble epoxide hydrolase inhibitor
}

Authors: Anamitra Ghosh ${ }^{1}$, Michele E. Comerota ${ }^{1}$, Debin Wan $^{2}$, Fading Chen ${ }^{1}$, Nicholas E. Propson ${ }^{1,3}$, Sung Hee Hwang ${ }^{2}$, Bruce D. Hammock ${ }^{2}$ and Hui Zheng ${ }^{1,3,4} *$

\author{
Affiliations: \\ ${ }^{1}$ Huffington Center on Aging, Baylor College of Medicine, Houston, TX \\ ${ }^{2}$ Department of Entomology and Nematology and UCDMC Comprehensive Cancer Center, University \\ of California, Davis, CA \\ ${ }^{3}$ Department of Molecular and Cellular Biology, Baylor College of Medicine, Houston, TX \\ ${ }^{4}$ Department of Molecular and Human Genetics, Baylor College of Medicine, Houston, TX
}

*To whom correspondence should be addressed: Hui Zheng, Email: huiz@bcm.edu

One Sentence Summary: We show that soluble epoxide hydrolase is upregulated in AD patients and mouse models, and that inhibition of this lipid metabolic pathway using an orally bioavailable small molecule inhibitor is effective in restoring brain epoxy fatty acids, ameliorating AD neuropathology and improving synaptic and cognitive function. 


\begin{abstract}
Neuroinflammation has been increasingly recognized to play critical roles in Alzheimer's disease (AD). The epoxy fatty acids (EpFAs) are derivatives of the arachidonic acid metabolism with antiinflammatory activities. However, their efficacy is limited due to the rapid hydrolysis by the soluble epoxide hydrolase $(\mathrm{sEH})$. We found that $\mathrm{sEH}$ is predominantly expressed in astrocytes where its levels are significantly elevated in postmortem human $\mathrm{AD}$ brains and in $\beta$-amyloid mouse models, and the latter is correlated with drastic reductions of brain EpFA levels. Using a highly potent and specific small molecule sEH inhibitor, 1-trifluoromethoxyphenyl-3-(1-propionylpiperidin-4-yl) urea (TPPU), we report here that TPPU treatment potently protected against LPS-induced inflammation in vitro and in vivo. Long-term administration of TPPU to the 5xFAD mouse model via drinking water reversed microglia and astrocyte reactivity and immune pathway dysregulation, and this is associated with reduced $\beta-$ amyloid pathology and improved synaptic integrity and cognitive function. Importantly, TPPU treatment reinstated and positively correlated EpFA levels in the 5xFAD mouse brain, demonstrating its brain penetration and target engagement. These findings support TPPU as a novel therapeutic target for the treatment of $\mathrm{AD}$ and related disorders.
\end{abstract}




\section{Introduction}

Alzheimer's disease (AD) is the most common form of age-associated neurodegenerative disorder and is an unmet medical need. AD is defined by the deposition of extracellular senile plaques composed of amyloid beta $(\mathrm{A} \beta)$ aggregates and the formation of intracellular neurofibrillary tangles containing abnormal hyperphosphorylated tau protein (1). Overwhelming evidence support the notion that the accumulation of $A \beta$ initiates a series of downstream events leading to cognitive impairment and neurodegeneration $(2,3)$. Hence, the majority of $\mathrm{AD}$ clinical trials have focused on reducing $\mathrm{A} \beta$ load. Unfortunately, these trials have been unsuccessful so far $(2,4-6)$. Thus, there is an urgent need to pursue other disease modifying therapies.

Besides the pathological hallmarks, $\mathrm{AD}$ is associated with prominent neuroinflammation $(7,8)$. Prolonged activation of glial cells, microglia and astrocytes in particular, and the release of proinflammatory cytokines, chemokines, and reactive oxygen and nitrogen species, create a neurotoxic environment which could exacerbate the progression of $\mathrm{AD}$ (9-11). Recent genome wide association studies have identified multiple immune related gene variants as risk factors for late-onset AD, supporting a major contributing role of innate immunity and neuroinflammation in AD (12-15). However, while epidemiological studies indicated positive effects for nonsteroidal anti-inflammatory drugs (NSAIDs) in AD development, randomized clinical trials failed to demonstrate clinical efficacy $(16,17)$.

Arachidonic acid (ARA) is an omega-6 unsaturated fatty acid present in the plasma membrane where it is bound to phospholipids (18). It can be released from the membrane by phospholipase A2 $\left(\mathrm{PLA}_{2}\right)$ and further metabolized by enzymes in three major pathways: cyclooxygenases (COXs), lipoxygenases (LOXs), and cytochrome P450 enzymes (CYPs), which produce prostaglandins, leukotrienes, and various eicosanoids including epoxyeicosatrienoic acids (EETs) (19, 20). Among these 
the COX and LOX pathways have been extensively studied and successfully targeted therapeutically (19). Of note, most of the NSAIDs are COX-1 and/or COX-2 inhibitors. In contrast, much less is known about the therapeutic potential of the CYP pathway.

Distinct from the well-established proinflammatory role of the prostaglandins, EETs and other epoxy fatty acids (EpFAs) have been proposed to possess anti-inflammatory properties $(21,22)$. However, they are broken down rapidly into their corresponding diols by the soluble epoxide hydrolase (sEH). Genetic deletion of Ephx2 (gene encoding sEH) or pharmacological inhibition of sEH conferred beneficial effects in several disease models, including depression, Parkinson's disease (23-26), and most recently, APP/PS1 transgenic mouse model of AD (27). However, these studies are restricted to acute model systems or germline deletions. It is not clear whether the sEH pathway can be therapeutically targeted under chronic conditions.

Here we present evidence that $\mathrm{sEH}$ is aberrantly elevated in the brain of $\mathrm{AD}$ individuals and $\mathrm{A} \beta$ mouse models, the latter is correlated with significant reduction of EpFA levels. Using a highly selective and potent sEH inhibitor, 1-trifluoromethoxyphenyl-3-(1-propionylpiperidin-4-yl) urea (TPPU) (23, 26), we show that long-term administration of TPPU to the 5xFAD mouse model restored the EpFA levels and reversed microglia and astrocyte reactivity and their associated molecular signatures. These are accompanied by attenuated $\beta$-amyloid pathology and improved synaptic integrity and cognitive function.

\section{Results}

\section{Elevated sEH and diminished EpFA levels associated with AD}

We first evaluated the expression of genes involved in arachidonic acid (ARA) metabolism in postmortem $\mathrm{AD}$ brain samples and their age-matched healthy controls (Fig. 1, A-B). Quantitative real- 
time PCR (qPCR) analysis showed that the expression of PLA2G2A which catalyzes the release of ARA from the membrane phospholipids, but not fatty acid amide hydrolase $(F A A H)$ which facilitates ARA production from endocannabinoids and degrades bioactive fatty acid amides, was significantly elevated, indicating that PLA2-mediated release of ARA is a major source for the upregulated ARA in AD. Both the $C O X-2$ and $C Y P 4 F 8$, which produce prostaglandin $(\mathrm{PG})$ and its metabolite $\mathrm{PGE}_{2}$, respectively, were significantly increased, suggesting overall activation of the cyclooxygenase metabolism. Interestingly, examination of the CYP monooxygenase pathway revealed that, while no differences in several of the CYPs, including CYP2J2, CYP2C8 and CYP2C19, were detected, expression of EPHX2 was significantly higher in AD samples compared to the controls (Fig. 1B). These results suggest aberrant regulation of the ARA metabolism in AD brains.

Consistent with the mRNA expression, Western blot analysis revealed a nearly two-fold increase of sEH protein in $\mathrm{AD}$ brains compared to controls (Fig. 1C and quantified in D). To substantiate these findings, we performed qPCR analysis of 5xFAD transgenic (Tg) mice, which showed increased Ephx2 expression in both the cortex and hippocampus at 4.5 months of age compared to their littermate nontransgenic (N-Tg) controls (Fig. 1E). Western blotting validated increased sEH levels in these mice (Fig. $1 \mathrm{~F}$ and quantified in G). Similar increases were also detected in an $\mathrm{APP}^{\mathrm{NLGF}}$ knock-in mice (28) with physiological expression of APP when compared with wild-type controls (fig. S1, A-B). The results combined demonstrate prominent upregulation of $\mathrm{sEH}$ in the brains of AD patients and APP/A $\beta$ mouse models.

Consistent with augmented sEH levels, LC-MS/MS-based lipidomic analysis of two major sEH eicosanoid substrates, EETs and epoxydocosapentaenoic acids (EDPs) showed dramatically reduced levels of multiple EET and EDP regioisomers in Tg brains in comparison to N-Tg controls (Fig. 1H). Similar to that of human samples, no alterations in the expression of CYP monooxygenases that produce 
EETs from the ARA, such as Cyp2c39, Cyp2j5 and Cyp2j9, were observed in Tg mice (fig. S1C). Thus, the reduced EETs and EDPs are likely due to specific increases of sEH activity downstream of CYP. These results suggest that elevated sEH plays an active role in hydrolyzing EETs and EDPs, leading to their reduced levels in $\mathrm{Tg}$, and possibly human $\mathrm{AD}$ brains.

\section{TPPU blocks astroglial sEH upregulation and LPS-induced inflammation}

To assess the cell type expression of $\mathrm{sEH}$, we used a flow cytometry-based concurrent brain cell type acquisition $(\mathrm{CoBrA})$ method to simultaneously isolate astrocytes, microglia and vascular endothelial cells (29). qPCR analysis of Ephx2 revealed that sEH is highly expressed in sorted astrocytes where its levels are significantly elevated compared to N-Tg controls (Fig. 2A). Interestingly, although modest and low levels of Ephx2 were detected in endothelial cells and microglia, respectively, there were no significant differences between $\mathrm{Tg}$ and $\mathrm{N}-\mathrm{Tg}$ controls. This result was substantiated by coimmunofluorescence staining of $\mathrm{sEH}$ with astrocyte (GFAP) or microglia (Iba-1) markers in hippocampal sections of $\mathrm{N}-\mathrm{Tg}$ and $\mathrm{Tg}$ brains (Fig. 2B). We observed elevated expression of sEH predominantly in GFAP-positive astrocytes in Tg samples, with negligible co-staining with Iba1, consistent with previous findings (27). Both the sEH fluorescence intensity and the number of sEH expressing cells were increased significantly in Tg mice compared to N-Tg controls (Fig. 2C). Similar astrocytic upregulation of sEH was also observed by acute LPS administration in vivo (fig.S2) and in primary astrocyte cultures (fig. S3, A-B), and this is associated with increased nitrite release as measured by the Griess assay (Fig. 2D) and the expression of proinflammatory cytokines (Fig. 2E).

Using these parameters, we tested the effect of sEH inhibitor TPPU in LPS-treated mouse primary astrocyte cultures. Thirty minutes pre-treatment of TPPU dose-dependently reduced nitrite release (Fig. 2D), and the expression of proinflammatory molecules $I l-1 \alpha, I l-1 \beta, T n f-\alpha, I l-6$, iNOS and 
Gfap (Fig. 2E) and $C c l-2$ and $C x c l-1$ (fig. S3C). The reduced expression of iNOS and GFAP were confirmed by immunostaining (Fig. 2, F-G). In agreement with the astroglial specific expression of sEH, TPPU failed to attenuate LPS-induced expression of pro-inflammatory molecules in primary microglia cultures (fig. S4).

\section{EETs are functional mediators of TPPU}

Next, we wondered whether the anti-inflammatory effect of sEH inhibition was attributed to increased EETs. We pre-treated primary astrocytes with TPPU and/or a putative pan-EET receptor antagonist 14,15-EEZE 30 minutes prior to LPS treatment and measured the nitrite levels by Griess assay (30). We observed that the inhibitory effect of TPPU was completely abolished upon co-treatment with 14,15EEZE (Fig. 2H), suggesting that EETs and possibly related EpFA are the functional lipid mediators of TPPU.

We then went on to test whether exogenous EET could directly mitigate LPS-induced inflammation. We found that 30-minute pre-treatment of 11,12-EET dose dependently attenuated the LPS-induced nitrite release in both cultured primary astrocytes (Fig. 2I) and microglia (Fig. 2J). EET treatment also suppressed the LPS-induced expression of pro-inflammatory molecules $I l-1 \alpha, I l-1 \beta, T n f$ $\alpha, I l-6, i N O S$ and $C 3$ in primary microglia cultures (fig. S5). The efficacy of EET in preventing LPSinduced cytokine expression was further validated using an ex vivo mouse hippocampal organotypic slice culture system (fig. S6). Collectively, these results support a pathway whereby TPPU inhibits astroglial sEH activity, and the resulting augmentation of EETs exert anti-inflammatory effect in both astrocytes and microglia through autocrine and paracrine activities.

\section{TPPU mitigates LPS-induced acute inflammation in vivo}


Given the strong anti-inflammatory effect of TPPU in vitro, we thought to assess its in vivo efficacy. We first examined whether TPPU is a substrate for P-glycoprotein (P-gp), which mediates the ATPdependent efflux of drugs or xenobiotics (31). Using the well-established human Caco-2 cells (32, 33), we determined that the apparent permeability coefficient $\left(P_{a p p}\right)$ for TPPU from basolateral to apical (B to A) was 24.45 and from A to B was 18.03 (Table S1). Thus, the B to A/A to B efflux ratio was 1.36, which was decreased to 0.99 in the presence of verapamil (a P-gp inhibitor). Since a ratio of >2 is generally considered to involve P-gp-mediated efflux (33), TPPU is unlikely to be a good P-gp substrate.

Next, we investigated the effect of TPPU in LPS-induced inflammation by pre-treating the C57BL/6 mice with one dose of TPPU ( $3 \mathrm{mg} / \mathrm{kg}$ ) via oral gavage for 24 hours, followed by co-treatment with LPS (3 mg/kg, i.p.) and TPPU (3 mg/kg, oral gavage), and the mice were euthanized after 18 hours (fig. S7A). Consistent with the in vitro and ex vivo studies, qPCR analysis of brain samples showed that LPS triggered the expression of proinflammatory molecules in both the cortex and hippocampus, and the vast majority of these were significantly reduced by TPPU (fig. S7B). Western blotting showed that levels of iNOS, COX-2, GFAP, Iba-1 and sEH were upregulated in LPS-treated mice but downregulated by TPPU in both the hippocampus and cortex (fig. S7, C-F). Immunofluorescence staining of Iba-1 and GFAP and co-staining with COX-2 and iNOS documented that TPPU mitigated LPS-induced microglia and astrocyte cell numbers and staining intensities, respectively, as well as COX-2 and iNOS expressions (fig. S8). Together, our results demonstrate that sEH blockade by TPPU prevents acute neuroinflammation in vitro and in vivo.

\section{TPPU enters the brain and engages its target under chronic treatment conditions}


Given the heightened expression of $\mathrm{sEH}$ in $\mathrm{AD}$ human brains and mouse models and the strong acute anti-inflammatory effect of TPPU, we thought to test the long-term therapeutic effect of TPPU in 5xFAD Tg mice. We supplied either Vehicle (Veh) or TPPU to Tg mice and their N-Tg littermate controls via drinking water starting at 2 months of age and continuously for 2.5 or 4.5 months (fig. S9A). Measurement of average water consumption per week per mouse for 10 weeks found no significant differences between Tg Veh and Tg TPPU groups, demonstrating that TPPU did not affect fluid intake (fig. S9B). Measurement of TPPU in brain and plasma samples showed that, whereas TPPU is undetectable in Veh-treated controls, its levels can be clearly measured both in the brain (fig. S9C) and plasma (fig. S9D); the resulting brain to plasma ratio is $21.7 \%$ and $17.2 \%$ for $\mathrm{N}-\mathrm{Tg}$ and $\mathrm{Tg}$ group, respectively, consistent with that of acute administration (26). These results establish that TPPU is able to gain access to the brain where its levels can be maintained under chronic treatment conditions.

To determine the target engagement of TPPU in the brain, we measured levels of EETs and EDPs, which were significantly reduced in Tg brains (Fig. 1H). We found that the EETs (Fig. 3A) and EDPs (Fig. 3B) were elevated in TPPU-treated Tg mice compared to the vehicle controls. The trending, but not statistically significant, increases of some of the regioisomers could be attributed to the differences in drug uptake or response among individual animals. Interestingly, plotting the coexpression relationship between EpFA regioisomers and TPPU observed prominent positive correlations between these two factors in the brain (Fig. 3, C-D), strengthening the notion that TPPU penetrates the brain and antagonizes its target sEH.

\section{TPPU treatment reverses immune pathway dysregulation in $\mathrm{Tg}$ mice}

Having established the pharmacodynamics of TPPU, we tested its effect in 5xFAD mice by employing molecular, biochemical, neuropathological and functional approaches as outlined (fig. S9A). To 
investigate the molecular mechanisms, we performed multiplex gene expression analysis using a Nanostring nCounter panel enriched for inflammatory genes. We quantified expression of 757 genes from the hippocampi of 4.5-month-old N-Tg and Tg mice treated with Veh or TPPU (n=4/group). Volcano plot representation of gene expression stratified by Veh-treated Tg vs N-Tg (Fig. 3E) demonstrated significant upregulation of 171 genes (red dots) in Tg mice. For each dot, significance is plotted against fold-change (log2 values). Treatment with TPPU for 2.5 months downregulated 73 inflammatory genes (green dots) compared to the Tg Veh group (Fig. 3F). Analysis of eigengene expression by heatmap in this inflammatory module for individual animals demonstrated that increased inflammatory gene expression in the Tg Veh was overall downregulated by TPPU (Fig. 3G). Next, we performed gene ontology (GO) enrichment analysis to gain further insights into the biological functions of differentially expressed genes (DEGs). Our results showed that 64 pathways were significantly enriched for the identified DEGs $(\mathrm{P}<0.05)$. DEGs that were mainly enriched in immune-related processes, such as immune responses, inflammatory responses, chemokine responses, cytokine mediated signaling pathway, iNOS biosynthetic process, complement activation pathway, LPS-mediated signaling pathway, cell adhesion and chemotaxis pathway, NF-кB mediated signaling pathway and apoptotic signaling pathways (Fig. 3H, Tg vs N-Tg). All the inflammatory pathways upregulated in Tg Veh mice were significantly downregulated with TPPU treatment (Fig. 3H, Tg TPPU vs Tg Veh). The Nanostring results were further validated by qPCR analysis of selected proinflammatory molecules using both cortex and hippocampal tissues (Fig. 3I).

In line with the gene expression data, Western blot analysis documented elevated levels of inflammatory (iNOS and COX-2) and glial cell (GFAP and Iba-1) markers in Tg mice compared to the N-Tg controls both in hippocampus (Fig. 4, A-B) and cortex (fig. S9, E-F). 2.5 months of TPPU treatment resulted in significant downregulation of these proteins. These results were further validated 
by immunofluorescence staining of Iba-1 (Fig. 4C) and GFAP (Fig. 4D) and co-staining with COX-2 and iNOS, followed by quantification of their intensities and the number of Iba-1 and GFAP positive cells (Fig. 4E). Together, the findings strongly suggest that inhibition of sEH by TPPU is effective in reversing the dysregulated immune pathways and glia reactivity in Tg mice.

\section{TPPU ameliorates A $\beta$ pathology and functional impairment}

Having demonstrated a significant role of TPPU in reversing AD-associated immune system dysfunction, we then asked whether sEH inhibition by TPPU may influence A $\beta$ pathology. We stained the brain sections of 4.5 months and 6.5 months Tg mice treated with Veh or TPPU and quantified the A $\beta$ plaque pathologies in the hippocampus (Fig. 5) and cortex (fig. S10). We observed modest 6E10positive $A \beta$ plaque deposition in 4.5 months Tg mice (Fig. 5A, Veh), which became more severe at 6.5 months (Fig. 5D, Veh). TPPU treatment led to significant reductions in the number, size, and intensities of $\mathrm{A} \beta$ plaques in both the 4.5 months (Fig. 5C) and the 6.5 months (Fig. 5F) groups. This was associated with reduced microglia activation surrounding A $\beta$ plaques, marked by CD68 staining (Fig. 5, B-C, E-F). The same results were obtained when cortical samples were analyzed (fig. S10).

We next assessed the role of TPPU in rescue of neuronal phenotypes. Immunostaining with presynaptic protein synaptophysin (Syp) and high-resolution imaging of 6.5-month-old Veh or TPPU treated $\mathrm{Tg}$ mice and $\mathrm{N}-\mathrm{Tg}$ controls observed significant reduction of Syn levels in area CA3 of hippocampus of Tg mice compared to N-Tg controls (Fig. 6A and quantified in B, Tg vs N-Tg, Veh). 4.5 months of TPPU treatment partially but significantly elevated the Syp expression (Fig. 6, A-B). LTP recordings of the Schaffer collateral pathway of the hippocampus revealed significant reductions in the Tg Veh group compared to N-Tg mice, and this phenotype was significantly improved in Tg TPPU mice (Fig. 6, C-D). Lastly, we evaluated the effect of TPPU in cognition using the novel object recognition 
(NOR) and fear conditioning (FC) paradigms (Fig. 6, E-F). The NOR assesses the hippocampus dependent long-term recognition memory by calculating the percent time spent with a novel object (object discrimination index, ODI). The Tg Veh mice displayed a significantly decreased ODI average, which was elevated significantly upon TPPU treatment (Fig. 6E). We further performed the FC paradigm to test hippocampal dependent (contextual test), and independent (cued test) associative learning (Fig. 6F). The four groups tested exhibited no differences in freezing percentage during the conditioning phase. During the context test, Tg vehicle mice displayed significantly decreased freezing percentage compared to N-Tg groups, suggesting an impaired contextual memory. Comparatively, the TPPU treated Tg mice exhibited significant increase in freezing frequency compared to the Tg Veh group. In addition, the percentage of freezing displayed post-cue was presented in the cued test was at similar levels between the groups. Thus, the $\mathrm{Tg}$ mice exhibit specific impairment in the hippocampus dependent contextual fear conditioning, and this phenotype is significantly improved by TPPU treatment. Taken together these results demonstrate that TPPU rescues synaptic deficits, LTP, and cognitive behaviors in $\mathrm{Tg}$ mice.

\section{Discussion}

Using postmortem human brain samples, primary cell cultures and AD mouse models, we investigated the role of sEH in neuroinflammation and $\mathrm{AD}$ pathogenesis and tested the therapeutic effect of an orally bioavailable small molecule sEH inhibitor, TPPU. We found that the sEH levels are elevated in human $\mathrm{AD}$ brains and $\mathrm{A} \beta$ mouse models, the latter is well-correlated with significantly lower levels of EETs and EDPs. Pre-treatment with EET and TPPU prevent acute LPS-induced neuroinflammation. Longterm TPPU treatment at the onset of AD neuropathology is able to reverse microglia and astrocyte activation and immune pathway dysregulation at the molecular, cellular and functional levels, and these 
are associated with attenuated $\mathrm{A} \beta$ pathology and improved synaptic and cognitive function. Moreover, TPPU reinstates and positively correlates the EpFA levels in the Tg brain, supporting its brain penetration and target engagement.

Upregulation of sEH expression has been reported in CNS disorders such as depression (26), schizophrenia (34), and Lewy body dementia and Parkinson's disease (25), and its inhibition has been shown to be beneficial in model systems. Lee et al (27) recently reported that sEH is upregulated in APP/PS1 mice. Our results clearly demonstrate that sEH is not only elevated in multiple transgenic AD mouse models, but also in human AD brains support the idea that it is a conserved and common feature in diseases with neuroinflammatory underpinning. Analysis of other molecules involved in ARA metabolism indicates partial activation of the ARA cascade. Interestingly, although the expression of $C O X-2$ and downstream $C Y P 4 F 8$ are both elevated, only EPHX2 but not CYP genes are altered. How this partial activation is achieved and whether this is also the case in other diseases is not understood. However, the data suggest that reduced EETs (and EDPs) are the result of their increased metabolism by sEH rather than insufficient conversion from ARA or release from phospholipids.

Our cell-type specific analysis demonstrates that astrocytes are the predominant cells expressing $\mathrm{sEH}$ where it is deregulated in AD conditions. This leads to diminished levels of EETs and EDPs and their anti-inflammatory activities on both astrocytes and microglia. Although no defined receptors have been identified for EETs, TRPV4 and G-proteins have been implicated in neuroinflammatory pathways downstream of EETs $(35,36)$. Besides the astrocytes, sEH is known to be highly expressed in the vasculature where it mediates vascular inflammation and barrier function through both EETs and EDPs $(21,37)$. Our expression analysis of sorted vascular endothelial cells revealed no appreciable differences in Ephx2 expression between the Tg mice and N-Tg controls, arguing against a major contribution of vascular sEH in disease pathogenesis. Nevertheless, it remains possible that the overall therapeutic 
effect of TPPU is due to its inhibition of sEH in both astrocytes and vascular endothelia, and possibly other cell types. Interestingly, a recent report (38) showed that the liver sEH modulates depressive behaviors in mice through central-peripheral interactions. As such, although our finding that TPPU enters the brain where its levels correlate with EETs and EDPs supports a CNS intrinsic mechanism, we cannot exclude the possibility that TPPU could also exert its effect through inhibition of liver sEH. The availability of the Ephx2 conditional allele (38) allows deciphering the cell-type specific effect and central- peripheral interactions.

EETs have been reported to act on multiple immune modulators, including the p38 MAP kinase, NF-кB, and STAT3 (39). EpFA are also known to stabilize mitochondria, reduce reactive oxygen species and shift the endoplasmic reticulum stress response from initiation of inflammation and cell death back towards maintaining cellular homeostasis (40). Thus, TPPU through EET and other EpFA could be acting on this pathway to reduce neuroinflammation. Of particular interest, we found in brain tissue as has been observed earlier in peripheral tissue that TPPU potently inhibits COX-2 expression (26), which has been widely implicated in AD. While the precise mechanism for this regulation remains to be established, it raises the intriguing possibility that TPPU's anti-inflammatory activity may be conferred through inhibition of both sEH and COX-2.

Consistent with the above studies, our Nanostring analysis followed by qPCR validation revealed multiple immune and inflammatory response pathways are upregulated in the $\mathrm{Tg}$ mice and downregulated by TPPU. In addition, recent reports identified a signaling pathway whereby microglia mediated neuroinflammation, in a C1q-, IL-1 $\alpha$ - and TNF-dependent manner, induces A1 astrocyte genes that are toxic to the neurons (41-43). Our gene expression analysis revealed that a number of A1 astrocyte genes (Serping1, Gbp2, Srgn, H2T23, Psmb8) were normalized by TPPU, suggesting that TPPU may reduce neuroinflammation via mitigating astrocytic activation, thus promoting neuronal 
survival. Additionally, the NLRP3 inflammasome activation has been implicated in producing harmful chronic inflammatory reactions and impairing microglial $\mathrm{A} \beta$ clearance and cognitive function in $\mathrm{AD}(8$, 44, 45). Indeed, we found that Nlrp3 and Caspl were both upregulated in Tg mice and downregulated by TPPU treatment. TPPU could suppress chronic neuroinflammation through NLRP3 dependent mechanisms.

We demonstrate that long-term TPPU treatment not only dampens glia reactivity but also ameliorates $\mathrm{A} \beta$ pathology and improves functional outcomes in Tg mice. Ample evidence documents that prolonged microglia activation leads to impaired $\mathrm{A} \beta$ phagocytosis and triggers the production of proinflammatory mediators, and its inhibition reverses these anomalies $(46,47)$. Therefore, the observed reduction of $A \beta$ pathology and improvement of neuronal function by TPPU could be the consequences of glia normalization. Additionally, microglia, through complement-dependent mechanisms, has been shown to mediate synapse elimination $(48,49)$. Since we observed robust rescue of aberrant $C 1 q$ and $C 3$ expression in Tg mice by TPPU (Fig. 3), the augmented synaptic protein expression and behavioral performance could be attributed by improved synapse maintenance.

Several classes of sEH inhibitors have been developed (50). Overall, they are well-tolerated in preclinical studies, establishing the large safety window for sEH targeting. Among these, TPPU is widely used as a tool compound because of its superior potency, specificity, and pharmacokinetics (23, 51-54). Of particular interest, a Phase 1 trial was recently initiated to test a TPPU analog (EC5026) for neuropathic pain (https://www.prnewswire.com/news-releases/eicosis-announces-first-subjectdosed-in-phase-1a-clinical-trial-of-ec5026-300971946.html). We report here that long-term administration of TPPU results in significant brain retention where it engages its target and affords beneficial effect in a mouse model of AD. These features make TPPU an attractive lead candidate for the treatment of $\mathrm{AD}$ and possibly other neurodegenerative diseases. 
bioRxiv preprint doi: https://doi.org/10.1101/2020.06.30.180984; this version posted July 2, 2020. The copyright holder for this preprint (which was not certified by peer review) is the author/funder. All rights reserved. No reuse allowed without permission. 


\section{Materials and Methods:}

\section{Study design}

The goal of this study was to establish evidence, using multiple model systems, that inhibition of the soluble epoxide hydrolase via TPPU is a viable approach to modify disease progression in AD. In the setting of experiment, one individual would randomize the animals, plates, and slides, and another would analyze them. The minimum sample size for all experiments was held at six mice per group based on the design of previous studies (11). To improve our power, and thus our ability to statistically detect smaller effects, many of our analyses included more mice per group. Further experimental details and protocols of each model, including animal care/handling and the number of biological/technical replicates, are in this section, and in Figure Legends.

\section{Human subjects}

Postmortem brain tissues were provided by the University of Pennsylvania Center for Neurodegenerative Disease Research (CNDR). Informed consent was obtained from all subjects. The demographic data can be found in Supplementary Table 2. Influence of sex, gender identity or both on the study results was not the objective of the study. It was not analyzed due to small sample size.

\section{Mice and treatment}

The C57BL/6, and 5xFAD mice were obtained from the Jackson Laboratory (Bar Harbor, ME). $\mathrm{APP}^{\mathrm{NLGF}}$ mice were obtained from RIKEN (28). Mice were housed 4-5 per cage in a pathogen free mouse facility with ad libitum access to food and water on a $12 \mathrm{hr}$ light/dark cycle. Male and female mice at approximately equal ratio were used unless otherwise specified. All procedures were performed in accordance with NIH guidelines and approval of the Baylor College of Medicine Institutional Animal Care and Use Committee (IACUC). 
In the acute TPPU regimen, ten- to twelve-week-old C57BL/6 mice received $1^{\text {st }}$ dose of TPPU (3 $\mathrm{mg} / \mathrm{kg}$ ) via oral gavage $24 \mathrm{~h}$ before co-treatment of LPS ( $3 \mathrm{mg} / \mathrm{kg}$, i.p. $)$ and TPPU ( $2^{\text {nd }}$ dose). Eighteenhour post co-treatment, mice were sacrificed for analysis. The sEH inhibitor TPPU was synthesized as previously described (23). TPPU was dissolved either in DMSO for in vitro and ex vivo treatment or in 10\% polyethylene glycol 400 (PEG400, Fisher) for in vivo treatment. The vehicle mice received oral gavage treatment of $1 \%$ PEG400. Mice were perfused with saline before collecting brain for biochemical analysis.

\section{Primary microglia and astrocyte cultures}

Primary glia cultures were prepared as described previously (48). In brief, mouse cortices and hippocampi were isolated from newborn pups (P0-P1) in dissection medium (HBSS with $10 \mathrm{mM}$ HEPES, $1 \%$ v/v Pen/Strep) and cut into small pieces. Tissue was digested with $2.5 \%$ trypsin at $37^{\circ} \mathrm{C}$ for $15 \mathrm{~min}$ before trypsin inhibitor $(1 \mathrm{mg} / \mathrm{ml})$ was added. Next, tissue was centrifuged for $5 \mathrm{~min}$ at 1500 rpm, triturated, and resuspended in DMEM medium with 10\% FBS. Cells were plated onto poly-Dlysine (PDL)-coated T-75 flasks at 50,000 cells $/ \mathrm{cm}^{2}$ to generate mixed glial cultures. When confluent, microglia were separated by tapping the flasks against table and collecting the floating cells in media. Microglia cells were then seeded at 50,000 cells $/ \mathrm{cm}^{2}$ and cultured for another day in PDL-coated 12well plates for mRNA assays or on coverslips for staining. After collecting microglia cells, remaining cells (mostly astrocytes) were trypsinized and seeded at 40,000 cells $/ \mathrm{cm}^{2}$ and cultured for another two days in PDL-coated plated for mRNA assays or immunocytochemistry.

\section{RNA extraction and expression analysis}

Total RNA was extracted from cells or human or mouse brain tissues using RNeasy Mini kit (Qiagen, 74106). Reverse transcription was carried out using iScript Reverse Transcription Supermix (Bio-Rad, 1708840). The qPCR analyses were performed using SYBR Green PCR master mix (Bio-Rad) on a 
CFX384 Touch Real-Time PCR Detection System. Primer sequences can be found in Supplementary Table 3 .

For Nanostring analysis, RNA was isolated from 4.5-month-old mouse hippocampus and 770 transcripts were quantified with the Nanostring nCounter multiplexed target platform using the Mouse Neuroinflammation panel (https://www.nanostring.com). nCounts of mRNA transcripts were normalized using the geometric means of 10 housekeeping genes (Csnk2a2, Ccdc127, Xpnpep1, Lars, Supt7l, Tada2b, Aars, Mto1, Tbp, and Fam104a) and analyzed using nSolver 4.0 and the Advanced Analysis 2.0 plugin. Fold-change expression and p-values were calculated by linear regression analysis using negative binomial or log-linear models. P-values were corrected for multiple comparisons using the Benjamini-Yekutieli method. Volcano plots of differential expression data were plotted using the $\log 10$ (p-value) and $\log 2$ fold-change using the Graphpad prism. Gene ontology enrichment analysis was performed using https://www.innatedb.com/. Heatmaps were constructed using Graphpad prism.

\section{Griess assay}

Griess assay was performed, as described previously (55). Briefly, standards in triplicate were used for each plate. Standard mix was made up of $1 \mathrm{ml}$ media and $1 \mu \mathrm{l}$ sodium nitrite and added to wells in volumes increasing by $5 \mu \mathrm{l}$ from 0-35 $\mu \mathrm{l}$. Media was added to each well to bring volume up to a total of $100 \mu \mathrm{l}$. Sample supernatant was added in duplicate to remaining wells in $100 \mu \mathrm{l}$. Then, $100 \mu \mathrm{l}$ of Griess reagent (Sigma) was added to each well and incubated at RT for 20 minutes. Absorbance at $540 \mathrm{~nm}$ was detected in a Synergy 2 Multi-Detection Microplate Reader.

\section{P-glycoprotein substrate evaluation}

Caco- 2 cells were diluted to $6.86 \times 10^{5}$ cells $/ \mathrm{mL}$ with culture medium and $50 \mu \mathrm{l}$ of cell suspension were dispensed into the filter well of the 96-well HTS Transwell plate. Cells were cultivated for 14-18 days in a cell culture incubator at $37^{\circ} \mathrm{C}, 5 \% \mathrm{CO}_{2}, 95 \%$ relative humidity. Electrical resistance was measured 
across the monolayer by using Millicell Epithelial Volt-Ohm measuring system. "TEER of each well is calculated by the equation- TEER value $\left(\mathrm{ohm} \bullet \mathrm{cm}^{2}\right)=$ TEER measurement (ohms) x Area of membrane $\left(\mathrm{cm}^{2}\right)$. The TEER value of each well should be greater than $230 \mathrm{ohms} \bullet \mathrm{cm}^{2}$. Digoxin was used as the reference substrate of P-gp. Propranolol was used as the high permeability marker. To determine the rate of drug transport in the apical to basolateral direction, working solutions containing TPPU was added to the Transwell insert (apical compartment). To determine the rate of drug transport in the basolateral to apical direction, working solutions containing TPPU was added to each well of the receiver plate. To determine the rate of drug transport in the presence of the P-gp inhibitor, verapamil the known inhibitor of Pgp, was added to both apical and basolateral compartments at a final concentration of $100 \mu \mathrm{M}$, followed by incubation at $37^{\circ} \mathrm{C}$ for 2 hours. Next, samples from apical and basolateral wells were transferred to a new 96-well plate and cold acetonitrile containing appropriate internal standards (IS) were added into each well of the plate(s). Samples were analyzed by an LC-MS/MS. Percent parent compounds remaining at each time point are estimated by determining the peak area ratios from extracted ion chromatograms. The apparent permeability coefficient $\left(\mathrm{P}_{\mathrm{app}}\right)$, in units of centimeter per second, using the following equation: $\mathrm{P}_{\mathrm{app}}=(\mathrm{VA} \times[\mathrm{drug}]$ acceptor $) /($ Area $\times$ Time $\times[\mathrm{drug}]$ initial, donor $)$, where VA is the volume (in $\mathrm{ml}$ ) in the acceptor well, area is the surface area of the membrane $(0.143$ $\mathrm{cm}^{2}$ for Transwell-96 Well Permeable Supports), and time is the total transport time in seconds. The efflux ratio was determined using the following equation: Efflux Ratio $=\mathrm{P}_{\mathrm{app}}(\mathrm{B}-\mathrm{A}) / \mathrm{P}_{\mathrm{app}}(\mathrm{A}-\mathrm{B})$, where $\mathrm{P}_{\mathrm{app}}$ (B-A) indicates the apparent permeability coefficient in basolateral to apical direction, and $\mathrm{P}_{\text {app }}(\mathrm{A}-\mathrm{B})$ indicates the apparent permeability coefficient in apical to basolateral direction. The recovery can be determined using following equation: Recovery $\%=(\mathrm{VA} \times[\mathrm{drug}]$ acceptor $+\mathrm{VD} \times[\mathrm{drug}] \mathrm{donor}) /(\mathrm{VD} \times[\mathrm{drug}]$ initial, donor $)$, where $\mathrm{VA}$ is the 
volume (in $\mathrm{ml})$ in the acceptor well $(0.235 \mathrm{ml}$ for $\mathrm{Ap} \rightarrow \mathrm{Bl}$ flux, and $0.075 \mathrm{ml}$ for $\mathrm{Bl} \rightarrow \mathrm{Ap})$, VD is the volume (in $\mathrm{ml})$ in the donor well $(0.075 \mathrm{ml}$ for $\mathrm{Ap} \rightarrow \mathrm{Bl}$ flux, and $0.235 \mathrm{ml}$ for $\mathrm{Bl} \rightarrow \mathrm{Ap})$.

\section{Cell-type purification and FACS sorting}

Mice were perfused with ice-cold PBS, adult mouse brains (whole brain minus cerebellum) were chopped and resuspended in $2.5 \mathrm{mls}$ of HBSS w/o $\mathrm{Ca}^{2+}$, and w/o $\mathrm{Mg}^{2+}$ containing activated papain and DNase. Cell type purification and FACS sorting were done as described (29). Briefly, brains were incubated at $37^{\circ} \mathrm{C}$, then triturated 4 times with a fire-polished glass Pasteur pipet. Next, samples were mixed with $\mathrm{HBSS}+(\mathrm{HBSS}+0.5 \% \mathrm{BSA}, 2 \mathrm{mM}$ EDTA) and centrifuged for 5 min. The pellet was resuspended in $1000 \mathrm{ml}$ of $\mathrm{HBSS}+$ and centrifuged for $15 \mathrm{sec}$ at room temperature. The supernatant was collected and was filtered through cell strainer (BD SKU 352340) and centrifuged for 5 min at $300 \mathrm{~g}$ at $4^{\circ} \mathrm{C}$. To remove myelin, the Miltenyi myelin removal beads were used according to the manufacturer's instructions (Miltenyi, 130-096-733). After that, cells were centrifuged at 300xG for 5 min at $4^{\circ} \mathrm{C}$. Next, the cells were resuspended in $1 \mathrm{ml}$ HBSS+ solution and passed through a LS column. The total effluent was then centrifuged for $5 \mathrm{~min}$ at $300 \mathrm{~g}$ at $4^{\circ} \mathrm{C}$ to pellet the cells. For the antibody staining, the cells were resuspended in HBSS+ solution and then stained for with CD45-BV421 (BD, 563890), CD11bFITC (BD, 553310) for microglia, ACSA-2-APC (Miltenyi, 130-102-315) for astrocytes, and cell viability blue fluorescent dye (Invitrogen, L23105). After FACS sorting, the cells were collected in Eppendorf tubes, centrifuged at $1500 \mathrm{rpm}$ for $5 \mathrm{~min}$, and resuspended in RLT buffer containing $1 \%$ BME for future qPCR analysis. The mRNA was extracted using the QIAGEN RNAEasy Micro kit (QIAGEN, 74004).

\section{Immunostaining and quantification}

Cells were fixed with $4 \%$ paraformaldehyde in $1 \mathrm{X}$ PBS for $15 \mathrm{~min}$ and processed for immunocytochemistry, as described previously (56). First, nonspecific sites were blocked with $0.2 \%$ 
bovine serum albumin, $0.5 \%$ Triton $\mathrm{X}-100$, and $0.05 \%$ Tween 20 in PBS for $1 \mathrm{~h}$ at room temperature. Cells were then incubated with different primary antibodies: sEH (1:1000, Dr. Bruce Hammock lab (26)); GFAP (1:1000, Millipore); iNOS (1:500, Thermo Fisher); Iba-1 (1:1000, Wako) and COX-2 (1:1000, Thermo Fisher) at $4^{\circ} \mathrm{C}$ overnight. Appropriate secondary antibodies (Alexa Fluor 488 or 555 or 647, Invitrogen) were used followed by incubation with DAPI to stain the nucleus. The coverslipcontaining stained cells were washed twice with PBS and mounted on slides. After immunofluorescent staining, five individual areas from each coverslip were imaged using 20x magnification under a Leica TCS confocal microscope. Mean intensity of fluorescence and number of immunoreactive cells were quantified using the Image $\mathbf{J}$ software (NIH).

Immunohistochemistry was performed on free-floating microtome-cut sections $(30 \mu \mathrm{m}$ in thickness), as described previously (55). Briefly, mice brains were post fixed in $4 \%$ paraformaldehyde overnight at $4 \mathrm{C}$ and transferred to $30 \%$ sucrose solution following perfusion with saline. Sections were incubated with different antibodies: sEH (1:1000, Dr. Bruce Hammock lab (26)); GFAP (1:1000, Millipore); iNOS (1:250, Thermo Fisher); Iba-1 (1:800, Wako or 1:500, Novus Biologicals), COX-2 (1:500, Thermo Fisher), CD68 (1:500, BioLegend), 6E10 (1:1000, BioLegend). Appropriate secondary antibodies (Alexa Fluor 488 or 594 or 647, Invitrogen) were used followed by incubation with DAPI. A total of three to four sections per brain containing the hippocampus and cortex and five to seven mice per group were stained with antibodies as mentioned above. After immunofluorescence staining, confocal images were captured and mean intensity of fluorescence and number of immunoreactive cells were quantified using the Image $J$ software $(\mathrm{NIH})$. For quantification of $6 \mathrm{E} 10$ in the mouse cortex and hippocampus, sections were scanned using an EVOS FL Auto system. Images were then analyzed by ImageJ and background was subtracted by the software for fluorescence images before quantification.

\section{Western blotting}


Cells or brain tissues were collected and resuspended in modified radioimmunoprecipitation (RIPA) assay buffer containing protease and phosphatase inhibitor mixture. Cell suspensions were sonicated after resuspension, whereas mouse brain tissues were homogenized, sonicated, and then centrifuged at $14,000 \mathrm{x}$ for $45 \mathrm{~min}$ at $4^{\circ} \mathrm{C}$, as described previously (57). Briefly, protein concentrations were estimated using a BCA kit (Thermo Fisher). Lysates were separated on 7.5\%-15\% SDS-polyacrylamide electrophoresis gels (Bio-Rad). After the separation, proteins were transferred to a nitrocellulose membrane, and nonspecific binding sites were blocked by treating with either Odyssey blocking buffer (LI-COR) or TBS with 5\% bovine serum albumin (BSA) followed by antibody incubation: sEH (1:300, Santa Cruz or 1:1000, Dr. Bruce Hammock's lab (26)); iNOS (1:1000, Cell signaling); GFAP (1:15000, EMD Millipore); Iba-1 (1:1200, Wako); Cox-2 (1:500, Thermo Fisher); and $\beta$-actin (1:10,000, Sigma). Secondary IR-680-conjugated goat anti-mouse or goat anti-rabbit (1:10,000; Molecular Probes), IRDye 800-conjugated donkey anti-rabbit or donkey anti goat (1:10,000, Rockland, PA, USA) were used. Western blot images were captured with a LI-COR Odyssey machine (LI-COR). The western blot bands were quantified using ImageJ software (NIH).

\section{Behavioral analysis}

The novel object recognition (NOR) protocol included three phases; habituation phase, a training phase and an object recognition phase. The habitation phase included of one session, 5 minutes in length, in which the animals were allowed to freely explore a small Plexiglas arena (measuring $22 \mathrm{~cm} \mathrm{x} 44 \mathrm{~cm}$ ) that was utilized in the training and testing phase. One day after habituation the animals underwent training. During the training phase, the animals were placed in the same arena with the addition of two identical objects. The animals were allowed to freely explore the objects for 5 minutes. 24 hours after the training phase, the test phase was initiated. During the testing phase, the animal was placed in the same arena with one object previously explored in the training phase, the familiar object, and one novel object 
differing in color and shape, but sharing a common size and volume. The animals were allowed to freely explore the objects for 5 minutes. ANY-Maze software was used to measure time spent exploring each object. Exploration of an object was defined by head orientation directed toward the object or physical contact with the object. The object discrimination ratio (ODR) was calculated by the following formula: ODR $=($ Time exploring specified object $) /($ time exploring novel object + time exploring familiar object $)$ $\mathrm{x}$ 100. The fear conditioning protocol involved a training phase, context test, and a cued test as previously described (58). During the training phase the mice were placed in the training chamber and allowed to freely explore the environment. At 3 minutes, an 80-dB white noise was presented (auditory conditioned stimulus (CS)) for 30 seconds. During the last 2 seconds of the auditory stimulus, the unconditioned stimulus (US), a foot shock (0.8 mA, 2 seconds), was administered. The CS and US were then presented a second time at the 5-minute mark of the training procedure. After the second presentation of the US, the mice stayed in the training chamber for an additional 2 minutes without additional stimulations. The animals were returned to their original housing cages. 24 hours after the training procedure, the context test was performed. The mice were returned to the same training chamber consisting of the same context as the first procedure (same geometric shape of chamber, lights, scents and auditory sounds) for 3 minutes with no presentations of US or CS. One hour later, the cue test is performed. The cue test chamber consisted of a different geometric shape, flooring, light brightness and scent compared to the previous chamber used for training. After 3 minutes in the chamber, the auditory stimulus was presented for 3 minutes. The software, FreezeFrame3 and FreezeView (San Diego Instruments) was used to record and analyze the percent freezing in each trial.

\section{Electrophysiology}

Field recordings of Schaffer collateral LTP was performed as described before (59). Briefly, brains were isolated from 6.5-7-month-old mice and cut into $400 \mathrm{mM}$ slices on a vibratome. Hippocampal slices 
were incubated for $1 \mathrm{~h}$ at room temperature and then transferred to a heated recording chamber filled with recording ACSF (125 mM NaCl, $2.5 \mathrm{mM} \mathrm{KCl}, 1.25 \mathrm{mM} \mathrm{NaH}_{2} \mathrm{PO}_{4}, 25 \mathrm{mM} \mathrm{NaHCO}, 1 \mathrm{mM}$ $\mathrm{MgCl}_{2}, 2 \mathrm{mM} \mathrm{CaCl}$, and $10 \mathrm{mM}$ glucose, saturated with $95 \% \mathrm{O}_{2}$ and $5 \% \mathrm{CO}_{2}$ ) maintained at $32^{\circ} \mathrm{C}$. Stimulation of Schaffer collaterals from the CA3 region was performed with bipolar electrodes, while borosilicate glass capillary pipettes filled with recording ACSF (resistances of 2-3.5 M $\Omega$ ) were used to record field excitatory postsynaptic potentials (fEPSPs) in the CA1 region. Signals were amplified using a MultiClamp 700 B amplifier (Axon), digitized using a Digidata 1440A (Axon) with a $2 \mathrm{kHz}$ low pass filter and a $3 \mathrm{~Hz}$ high pass filter and then captured and stored using Clampex 10.4 software (Axon) for offline data analysis. The genotypes and treatment groups were blinded to the experimenter. For each experiment 10-13 sections from 5-6 animals per group/genotype were used.

\section{Measurement of brain and plasma drug concentrations}

$5 x F A D$ mice were treated with TPPU $(3 \mathrm{mg} / \mathrm{kg})$ in drinking water for 4 months and then euthanized. Plasma and whole-brain homogenates were extracted and subjected to LC-MS/MS analysis of TPPU and oxylipin on a 4000 Qtrap LC-MS/MS instrument (Applied Biosystems Instrument Corporation). For drug analysis in plasma, $10 \mu \mathrm{l}$ of plasma samples were transferred to $1.5 \mu 1$ Eppendorf tubes containing $90 \mu \mathrm{l}$ EDTA solution $(0.1 \%$ EDTA and $0.1 \%$ acetic acid), spiked with $10 \mu \mathrm{l}$ of $1 \mu \mathrm{g} / \mathrm{ml}$ TPPU-d3 in methanol, and subsequently subjected to liquid-liquid extraction by ethyl acetate (200 $\mu 1)$ twice (54). TPPU-d3 was added in each sample as an internal standard solution. The collected extraction solutions were dried using a speed vacuum concentrator, reconstituted in $50 \mu \mathrm{l}$ of $100 \mathrm{nM}$ CUDA in methanol, and ready for LC-MS/MS analysis. TPPU and oxylipin in brain tissues were analyzed simultaneously by a modified LC-MS/MS method(60) to including MRM transition of TPPU. Tissues ( 50 mg) were homogenized in ice cold methanol with $0.1 \%$ BHT and $0.1 \%$ acetic acid. The homogenates were spiked with $10 \mu \mathrm{l}$ of internal standard solution (mixture of deuterated compounds) and stored at $-80^{\circ} \mathrm{C}$ for $20 \mathrm{hr}$. 
After that, the homogenates were extracted using solid-phase extraction (Oasis-HLB Cartridge, Waters).

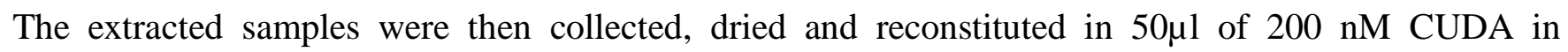
methanol. The analytes were then detected by the modified LC-MS/MS method.

\section{Quantification and statistical analysis}

All data were analyzed with GraphPad Prism v.7.04 and presented as mean \pm SEM $(* \mathrm{P}<0.05$, **P $<$ $0.01, * * * \mathrm{P}<0.001$ and $* * * * \mathrm{P}<0.0001)$. For simple comparisons, Student's t test was used. For multiple comparisons, ANOVA followed by the appropriate post hoc testing was utilized and is specified for each experiment in the figure legends. The statistical tests used for human data expression analysis is specified in the human data analysis methods section. All samples or animals were included in the statistical analysis unless otherwise specified. 


\section{References:}

1. H. W. Querfurth, F. M. LaFerla, Alzheimer's disease. N Engl J Med 362, 329-344 (2010).

2. J. M. Long, D. M. Holtzman, Alzheimer Disease: An Update on Pathobiology and Treatment Strategies. Cell 179, 312-339 (2019).

3. L. Mucke, D. J. Selkoe, Neurotoxicity of amyloid beta-protein: synaptic and network dysfunction. Cold Spring Harb Perspect Med 2, a006338 (2012).

4. J. L. Cummings, S. Cohen, C. H. van Dyck, M. Brody, C. Curtis, W. Cho, M. Ward, M. Friesenhahn, C. Rabe, F. Brunstein, A. Quartino, L. A. Honigberg, R. N. Fuji, D. Clayton, D. Mortensen, C. Ho, R. Paul, ABBY: A phase 2 randomized trial of crenezumab in mild to moderate Alzheimer disease. Neurology 90, e1889-e1897 (2018).

5. J. L. Cummings, T. Morstorf, K. Zhong, Alzheimer's disease drug-development pipeline: few candidates, frequent failures. Alzheimers Res Ther 6, 37 (2014).

6. D. R. Elmaleh, M. R. Farlow, P. S. Conti, R. G. Tompkins, L. Kundakovic, R. E. Tanzi, Developing Effective Alzheimer's Disease Therapies: Clinical Experience and Future Directions. J Alzheimers Dis 71, 715-732 (2019).

7. M. V. Guillot-Sestier, K. R. Doty, T. Town, Innate Immunity Fights Alzheimer's Disease. Trends Neurosci 38, 674-681 (2015).

8. M. T. Heneka, M. P. Kummer, A. Stutz, A. Delekate, S. Schwartz, A. Vieira-Saecker, A. Griep, D. Axt, A. Remus, T. C. Tzeng, E. Gelpi, A. Halle, M. Korte, E. Latz, D. T. Golenbock, NLRP3 is activated in Alzheimer's disease and contributes to pathology in APP/PS1 mice. Nature 493, 674-678 (2013).

9. M. T. Heneka, M. P. Kummer, E. Latz, Innate immune activation in neurodegenerative disease. Nat Rev Immunol 14, 463-477 (2014).

10. S. Prokop, K. R. Miller, F. L. Heppner, Microglia actions in Alzheimer's disease. Acta Neuropathol 126, 461-477 (2013).

11. H. Lian, H. Zheng, Signaling pathways regulating neuron-glia interaction and their implications in Alzheimer's disease. J Neurochem 136, 475-491 (2016).

12. B. W. Kunkle, B. Grenier-Boley, R. Sims, J. C. Bis, V. Damotte, A. C. Naj, A. Boland, M. Vronskaya, S. J. van der Lee, A. Amlie-Wolf, C. Bellenguez, A. Frizatti, V. Chouraki, E. R. Martin, K. Sleegers, N. Badarinarayan, J. Jakobsdottir, K. L. Hamilton-Nelson, S. Moreno-Grau, R. Olaso, R. Raybould, Y. Chen, A. B. Kuzma, M. Hiltunen, T. Morgan, S. Ahmad, B. N. Vardarajan, J. Epelbaum, P. Hoffmann, M. Boada, G. W. Beecham, J. G. Garnier, D. Harold, A. L. Fitzpatrick, O. Valladares, M. L. Moutet, A. Gerrish, A. V. Smith, L. Qu, D. Bacq, N. Denning, X. Jian, Y. Zhao, M. Del Zompo, N. C. Fox, S. H. Choi, I. Mateo, J. T. Hughes, H. H. Adams, J. Malamon, F. Sanchez-Garcia, Y. Patel, J. A. Brody, B. A. Dombroski, M. C. D. Naranjo, M. Daniilidou, G. Eiriksdottir, S. Mukherjee, D. Wallon, J. Uphill, T. Aspelund, L. B. Cantwell, F. Garzia, D. Galimberti, E. Hofer, M. Butkiewicz, B. Fin, E. Scarpini, C. Sarnowski, W. S. Bush, S. Meslage, J. Kornhuber, C. C. White, Y. Song, R. C. Barber, S. Engelborghs, S. Sordon, D. Voijnovic, P. M. Adams, R. Vandenberghe, M. Mayhaus, L. A. Cupples, M. S. Albert, P. P. De Deyn, W. Gu, J. J. Himali, D. Beekly, A. Squassina, A. M. Hartmann, A. Orellana, D. Blacker, E. Rodriguez-Rodriguez, S. Lovestone, M. E. Garcia, R. S. Doody, C. Munoz-Fernadez, R. Sussams, H. Lin, T. J. Fairchild, Y. A. Benito, C. Holmes, H. KaramujicComic, M. P. Frosch, H. Thonberg, W. Maier, G. Roshchupkin, B. Ghetti, V. Giedraitis, A. Kawalia, S. Li, R. M. Huebinger, L. Kilander, S. Moebus, I. Hernandez, M. I. Kamboh, R. Brundin, J. Turton, Q. Yang, M. J. Katz, L. Concari, J. Lord, A. S. Beiser, C. D. Keene, S. 
Helisalmi, I. Kloszewska, W. A. Kukull, A. M. Koivisto, A. Lynch, L. Tarraga, E. B. Larson, A. Haapasalo, B. Lawlor, T. H. Mosley, R. B. Lipton, V. Solfrizzi, M. Gill, W. T. Longstreth, Jr., T. J. Montine, V. Frisardi, M. Diez-Fairen, F. Rivadeneira, R. C. Petersen, V. Deramecourt, I. Alvarez, F. Salani, A. Ciaramella, E. Boerwinkle, E. M. Reiman, N. Fievet, J. I. Rotter, J. S. Reisch, O. Hanon, C. Cupidi, A. G. Andre Uitterlinden, D. R. Royall, C. Dufouil, R. G. Maletta, I. de Rojas, M. Sano, A. Brice, R. Cecchetti, P. S. George-Hyslop, K. Ritchie, M. Tsolaki, D. W. Tsuang, B. Dubois, D. Craig, C. K. Wu, H. Soininen, D. Avramidou, R. L. Albin, L. Fratiglioni, A. Germanou, L. G. Apostolova, L. Keller, M. Koutroumani, S. E. Arnold, F. Panza, O. Gkatzima, S. Asthana, D. Hannequin, P. Whitehead, C. S. Atwood, P. Caffarra, H. Hampel, I. Quintela, A. Carracedo, L. Lannfelt, D. C. Rubinsztein, L. L. Barnes, F. Pasquier, L. Frolich, S. Barral, B. McGuinness, T. G. Beach, J. A. Johnston, J. T. Becker, P. Passmore, E. H. Bigio, J. M. Schott, T. D. Bird, J. D. Warren, B. F. Boeve, M. K. Lupton, J. D. Bowen, P. Proitsi, A. Boxer, J. F. Powell, J. R. Burke, J. S. K. Kauwe, J. M. Burns, M. Mancuso, J. D. Buxbaum, U. Bonuccelli, N. J. Cairns, A. McQuillin, C. Cao, G. Livingston, C. S. Carlson, N. J. Bass, C. M. Carlsson, J. Hardy, R. M. Carney, J. Bras, M. M. Carrasquillo, R. Guerreiro, M. Allen, H. C. Chui, E. Fisher, C. Masullo, E. A. Crocco, C. DeCarli, G. Bisceglio, M. Dick, L. Ma, R. Duara, N. R. Graff-Radford, D. A. Evans, A. Hodges, K. M. Faber, M. Scherer, K. B. Fallon, M. Riemenschneider, D. W. Fardo, R. Heun, M. R. Farlow, H. Kolsch, S. Ferris, M. Leber, T. M. Foroud, I. Heuser, D. R. Galasko, I. Giegling, M. Gearing, M. Hull, D. H. Geschwind, J. R. Gilbert, J. Morris, R. C. Green, K. Mayo, J. H. Growdon, T. Feulner, R. L. Hamilton, L. E. Harrell, D. Drichel, L. S. Honig, T. D. Cushion, M. J. Huentelman, P. Hollingworth, C. M. Hulette, B. T. Hyman, R. Marshall, G. P. Jarvik, A. Meggy, E. Abner, G. E. Menzies, L. W. Jin, G. Leonenko, L. M. Real, G. R. Jun, C. T. Baldwin, D. Grozeva, A. Karydas, G. Russo, J. A. Kaye, R. Kim, F. Jessen, N. W. Kowall, B. Vellas, J. H. Kramer, E. Vardy, F. M. LaFerla, K. H. Jockel, J. J. Lah, M. Dichgans, J. B. Leverenz, D. Mann, A. I. Levey, S. Pickering-Brown, A. P. Lieberman, N. Klopp, K. L. Lunetta, H. E. Wichmann, C. G. Lyketsos, K. Morgan, D. C. Marson, K. Brown, F. Martiniuk, C. Medway, D. C. Mash, M. M. Nothen, E. Masliah, N. M. Hooper, W. C. McCormick, A. Daniele, S. M. McCurry, A. Bayer, A. N. McDavid, J. Gallacher, A. C. McKee, H. van den Bussche, M. Mesulam, C. Brayne, B. L. Miller, S. Riedel-Heller, C. A. Miller, J. W. Miller, A. Al-Chalabi, J. C. Morris, C. E. Shaw, A. J. Myers, J. Wiltfang, S. O'Bryant, J. M. Olichney, V. Alvarez, J. E. Parisi, A. B. Singleton, H. L. Paulson, J. Collinge, W. R. Perry, S. Mead, E. Peskind, D. H. Cribbs, M. Rossor, A. Pierce, N. S. Ryan, W. W. Poon, B. Nacmias, H. Potter, S. Sorbi, J. F. Quinn, E. Sacchinelli, A. Raj, G. Spalletta, M. Raskind, C. Caltagirone, P. Bossu, M. D. Orfei, B. Reisberg, R. Clarke, C. Reitz, A. D. Smith, J. M. Ringman, D. Warden, E. D. Roberson, G. Wilcock, E. Rogaeva, A. C. Bruni, H. J. Rosen, M. Gallo, R. N. Rosenberg, Y. Ben-Shlomo, M. A. Sager, P. Mecocci, A. J. Saykin, P. Pastor, M. L. Cuccaro, J. M. Vance, J. A. Schneider, L. S. Schneider, S. Slifer, W. W. Seeley, A. G. Smith, J. A. Sonnen, S. Spina, R. A. Stern, R. H. Swerdlow, M. Tang, R. E. Tanzi, J. Q. Trojanowski, J. C. Troncoso, V. M. Van Deerlin, L. J. Van Eldik, H. V. Vinters, J. P. Vonsattel, S. Weintraub, K. A. Welsh-Bohmer, K. C. Wilhelmsen, J. Williamson, T. S. Wingo, R. L. Woltjer, C. B. Wright, C. E. Yu, L. Yu, Y. Saba, A. Pilotto, M. J. Bullido, O. Peters, P. K. Crane, D. Bennett, P. Bosco, E. Coto, V. Boccardi, P. L. De Jager, A. Lleo, N. Warner, O. L. Lopez, M. Ingelsson, P. Deloukas, C. Cruchaga, C. Graff, R. Gwilliam, M. Fornage, A. M. Goate, P. Sanchez-Juan, P. G. Kehoe, N. Amin, N. Ertekin-Taner, C. Berr, S. Debette, S. Love, L. J. Launer, S. G. Younkin, J. F. Dartigues, C. Corcoran, M. A. Ikram, D. W. Dickson, G. Nicolas, D. Campion, J. Tschanz, H. Schmidt, H. Hakonarson, J. Clarimon, R. Munger, R. Schmidt, L. A. Farrer, C. Van 
Broeckhoven, C. O. D. M, A. L. DeStefano, L. Jones, J. L. Haines, J. F. Deleuze, M. J. Owen, V. Gudnason, R. Mayeux, V. Escott-Price, B. M. Psaty, A. Ramirez, L. S. Wang, A. Ruiz, C. M. van Duijn, P. A. Holmans, S. Seshadri, J. Williams, P. Amouyel, G. D. Schellenberg, J. C. Lambert, M. A. Pericak-Vance, Genetic meta-analysis of diagnosed Alzheimer's disease identifies new risk loci and implicates Abeta, tau, immunity and lipid processing. Nat Genet 51, 414-430 (2019).

13. C. Liu, J. Chyr, W. Zhao, Y. Xu, Z. Ji, H. Tan, C. Soto, X. Zhou, Genome-Wide Association and Mechanistic Studies Indicate That Immune Response Contributes to Alzheimer's Disease Development. Front Genet 9, 410 (2018).

14. R. Guerreiro, J. Bras, J. Hardy, SnapShot: genetics of Alzheimer's disease. Cell 155, 968968.e961 (2013).

15. D. Felsky, T. Roostaei, K. Nho, S. L. Risacher, E. M. Bradshaw, V. Petyuk, J. A. Schneider, A. Saykin, D. A. Bennett, P. L. De Jager, Neuropathological correlates and genetic architecture of microglial activation in elderly human brain. Nat Commun 10, 409 (2019).

16. C. H. Trepanier, N. W. Milgram, Neuroinflammation in Alzheimer's disease: are NSAIDs and selective COX-2 inhibitors the next line of therapy? J Alzheimers Dis 21, 1089-1099 (2010).

17. I. Cacciatore, L. Marinelli, E. Fornasari, L. S. Cerasa, P. Eusepi, H. Turkez, C. Pomilio, M. Reale, C. D'Angelo, E. Costantini, A. Di Stefano, Novel NSAID-Derived Drugs for the Potential Treatment of Alzheimer's Disease. Int J Mol Sci 17, (2016).

18. S. I. Rapoport, Arachidonic acid and the brain. J Nutr 138, 2515-2520 (2008).

19. H. C. Shen, B. D. Hammock, Discovery of inhibitors of soluble epoxide hydrolase: a target with multiple potential therapeutic indications. J Med Chem 55, 1789-1808 (2012).

20. J. W. Phillis, L. A. Horrocks, A. A. Farooqui, Cyclooxygenases, lipoxygenases, and epoxygenases in CNS: their role and involvement in neurological disorders. Brain Res Rev 52, 201-243 (2006).

21. K. Node, Y. Huo, X. Ruan, B. Yang, M. Spiecker, K. Ley, D. C. Zeldin, J. K. Liao, Antiinflammatory properties of cytochrome P450 epoxygenase-derived eicosanoids. Science 285, 1276-1279 (1999).

22. I. P. Koerner, R. Jacks, A. E. DeBarber, D. Koop, P. Mao, D. F. Grant, N. J. Alkayed, Polymorphisms in the human soluble epoxide hydrolase gene EPHX2 linked to neuronal survival after ischemic injury. J Neurosci 27, $4642-4649$ (2007).

23. T. E. Rose, C. Morisseau, J. Y. Liu, B. Inceoglu, P. D. Jones, J. R. Sanborn, B. D. Hammock, 1Aryl-3-(1-acylpiperidin-4-yl)urea inhibitors of human and murine soluble epoxide hydrolase: structure-activity relationships, pharmacokinetics, and reduction of inflammatory pain. $J$ Med Chem 53, 7067-7075 (2010).

24. H. J. Huang, Y. T. Wang, H. C. Lin, Y. H. Lee, A. M. Lin, Soluble Epoxide Hydrolase Inhibition Attenuates MPTP-Induced Neurotoxicity in the Nigrostriatal Dopaminergic System: Involvement of alpha-Synuclein Aggregation and ER Stress. Mol Neurobiol 55, 138-144 (2018).

25. Q. Ren, M. Ma, J. Yang, R. Nonaka, A. Yamaguchi, K. I. Ishikawa, K. Kobayashi, S. Murayama, S. H. Hwang, S. Saiki, W. Akamatsu, N. Hattori, B. D. Hammock, K. Hashimoto, Soluble epoxide hydrolase plays a key role in the pathogenesis of Parkinson's disease. Proc Natl Acad Sci U S A 115, E5815-e5823 (2018).

26. Q. Ren, M. Ma, T. Ishima, C. Morisseau, J. Yang, K. M. Wagner, J. C. Zhang, C. Yang, W. Yao, C. Dong, M. Han, B. D. Hammock, K. Hashimoto, Gene deficiency and pharmacological inhibition of soluble epoxide hydrolase confers resilience to repeated social defeat stress. Proc Natl Acad Sci U S A 113, E1944-1952 (2016). 
27. H. T. Lee, K. I. Lee, C. H. Chen, T. S. Lee, Genetic deletion of soluble epoxide hydrolase delays the progression of Alzheimer's disease. J Neuroinflammation 16, 267 (2019).

28. T. Saito, Y. Matsuba, N. Mihira, J. Takano, P. Nilsson, S. Itohara, N. Iwata, T. C. Saido, Single App knock-in mouse models of Alzheimer's disease. Nat Neurosci 17, 661-663 (2014).

29. D. B. Swartzlander, N. E. Propson, E. R. Roy, T. Saito, T. Saido, B. Wang, H. Zheng, Concurrent cell type-specific isolation and profiling of mouse brains in inflammation and Alzheimer's disease. JCI Insight 3, (2018).

30. T. H. Hung, S. K. Shyue, C. H. Wu, C. C. Chen, C. C. Lin, C. F. Chang, S. F. Chen, Deletion or inhibition of soluble epoxide hydrolase protects against brain damage and reduces microgliamediated neuroinflammation in traumatic brain injury. Oncotarget 8, 103236-103260 (2017).

31. M. F. Rosenberg, G. Velarde, R. C. Ford, C. Martin, G. Berridge, I. D. Kerr, R. Callaghan, A. Schmidlin, C. Wooding, K. J. Linton, C. F. Higgins, Repacking of the transmembrane domains of P-glycoprotein during the transport ATPase cycle. Embo j 20, 5615-5625 (2001).

32. P. V. Balimane, Y. H. Han, S. Chong, Current industrial practices of assessing permeability and P-glycoprotein interaction. Aaps j 8, E1-13 (2006).

33. R. Elsby, D. D. Surry, V. N. Smith, A. J. Gray, Validation and application of Caco-2 assays for the in vitro evaluation of development candidate drugs as substrates or inhibitors of Pglycoprotein to support regulatory submissions. Xenobiotica 38, 1140-1164 (2008).

34. M. Ma, Q. Ren, J. Yang, K. Zhang, Z. Xiong, T. Ishima, Y. Pu, S. H. Hwang, M. Toyoshima, Y. Iwayama, Y. Hisano, T. Yoshikawa, B. D. Hammock, K. Hashimoto, Key role of soluble epoxide hydrolase in the neurodevelopmental disorders of offspring after maternal immune activation. Proc Natl Acad Sci U S A 116, 7083-7088 (2019).

35. S. Cao, A. Anishkin, N. S. Zinkevich, Y. Nishijima, A. Korishettar, Z. Wang, J. Fang, D. A. Wilcox, D. X. Zhang, Transient receptor potential vanilloid 4 (TRPV4) activation by arachidonic acid requires protein kinase A-mediated phosphorylation. J Biol Chem 293, 5307-5322 (2018).

36. H. Watanabe, J. Vriens, J. Prenen, G. Droogmans, T. Voets, B. Nilius, Anandamide and arachidonic acid use epoxyeicosatrienoic acids to activate TRPV4 channels. Nature 424, 434438 (2003).

37. J. Hu, S. Dziumbla, J. Lin, S. I. Bibli, S. Zukunft, J. de Mos, K. Awwad, T. Fromel, A. Jungmann, K. Devraj, Z. Cheng, L. Wang, S. Fauser, C. G. Eberhart, A. Sodhi, B. D. Hammock, S. Liebner, O. J. Muller, C. Glaubitz, H. P. Hammes, R. Popp, I. Fleming, Inhibition of soluble epoxide hydrolase prevents diabetic retinopathy. Nature 552, 248-252 (2017).

38. X. H. Qin, Z. Wu, J. H. Dong, Y. N. Zeng, W. C. Xiong, C. Liu, M. Y. Wang, M. Z. Zhu, W. J. Chen, Y. Zhang, Q. Y. Huang, X. H. Zhu, Liver Soluble Epoxide Hydrolase Regulates Behavioral and Cellular Effects of Chronic Stress. Cell Rep 29, 3223-3234.e3226 (2019).

39. L. Williams, L. Bradley, A. Smith, B. Foxwell, Signal transducer and activator of transcription 3 is the dominant mediator of the anti-inflammatory effects of IL-10 in human macrophages. $J$ Immunol 172, 567-576 (2004).

40. K. M. Wagner, C. B. McReynolds, W. K. Schmidt, B. D. Hammock, Soluble epoxide hydrolase as a therapeutic target for pain, inflammatory and neurodegenerative diseases. Pharmacol Ther 180, 62-76 (2017).

41. S. A. Liddelow, K. A. Guttenplan, L. E. Clarke, F. C. Bennett, C. J. Bohlen, L. Schirmer, M. L. Bennett, A. E. Munch, W. S. Chung, T. C. Peterson, D. K. Wilton, A. Frouin, B. A. Napier, N. Panicker, M. Kumar, M. S. Buckwalter, D. H. Rowitch, V. L. Dawson, T. M. Dawson, B. Stevens, B. A. Barres, Neurotoxic reactive astrocytes are induced by activated microglia. Nature 541, 481-487 (2017). 
42. L. E. Clarke, S. A. Liddelow, Neurobiology: Diversity reaches the stars. Nature 548, 396-397 (2017).

43. Y. Shi, K. Yamada, S. A. Liddelow, S. T. Smith, L. Zhao, W. Luo, R. M. Tsai, S. Spina, L. T. Grinberg, J. C. Rojas, G. Gallardo, K. Wang, J. Roh, G. Robinson, M. B. Finn, H. Jiang, P. M. Sullivan, C. Baufeld, M. W. Wood, C. Sutphen, L. McCue, C. Xiong, J. L. Del-Aguila, J. C. Morris, C. Cruchaga, A. M. Fagan, B. L. Miller, A. L. Boxer, W. W. Seeley, O. Butovsky, B. A. Barres, S. M. Paul, D. M. Holtzman, ApoE4 markedly exacerbates tau-mediated neurodegeneration in a mouse model of tauopathy. Nature 549, 523-527 (2017).

44. D. Tejera, D. Mercan, J. M. Sanchez-Caro, M. Hanan, D. Greenberg, H. Soreq, E. Latz, D. Golenbock, M. T. Heneka, Systemic inflammation impairs microglial Abeta clearance through NLRP3 inflammasome. Embo j 38, e101064 (2019).

45. M. H. Park, M. Lee, G. Nam, M. Kim, J. Kang, B. J. Choi, M. S. Jeong, K. H. Park, W. H. Han, E. Tak, M. S. Kim, J. Lee, Y. Lin, Y. H. Lee, I. S. Song, M. K. Choi, J. Y. Lee, H. K. Jin, J. S. Bae, M. H. Lim, N,N'-Diacetyl-p-phenylenediamine restores microglial phagocytosis and improves cognitive defects in Alzheimer's disease transgenic mice. Proc Natl Acad Sci U S A 116, 23426-23436 (2019).

46. M. W. Salter, B. Stevens, Microglia emerge as central players in brain disease. Nat Med 23, 1018-1027 (2017).

47. H. Sarlus, M. T. Heneka, Microglia in Alzheimer's disease. J Clin Invest 127, 3240-3249 (2017).

48. A. Litvinchuk, Y. W. Wan, D. B. Swartzlander, F. Chen, A. Cole, N. E. Propson, Q. Wang, B. Zhang, Z. Liu, H. Zheng, Complement C3aR Inactivation Attenuates Tau Pathology and Reverses an Immune Network Deregulated in Tauopathy Models and Alzheimer's Disease. Neuron 100, 1337-1353.e1335 (2018).

49. Q. Shi, S. Chowdhury, R. Ma, K. X. Le, S. Hong, B. J. Caldarone, B. Stevens, C. A. Lemere, Complement C3 deficiency protects against neurodegeneration in aged plaque-rich APP/PS1 mice. Sci Transl Med 9, (2017).

50. S. Zarriello, J. P. Tuazon, S. Corey, S. Schimmel, M. Rajani, A. Gorsky, D. Incontri, B. D. Hammock, C. V. Borlongan, Humble beginnings with big goals: Small molecule soluble epoxide hydrolase inhibitors for treating CNS disorders. Prog Neurobiol 172, 23-39 (2019).

51. A. Ulu, S. Appt, C. Morisseau, S. H. Hwang, P. D. Jones, T. E. Rose, H. Dong, J. Lango, J. Yang, H. J. Tsai, C. Miyabe, C. Fortenbach, M. R. Adams, B. D. Hammock, Pharmacokinetics and in vivo potency of soluble epoxide hydrolase inhibitors in cynomolgus monkeys. $\mathrm{Br} \mathrm{J}$ Pharmacol 165, 1401-1412 (2012).

52. Y. Liu, X. Lu, S. Nguyen, J. L. Olson, H. K. Webb, D. L. Kroetz, Epoxyeicosatrienoic acids prevent cisplatin-induced renal apoptosis through a p38 mitogen-activated protein kinaseregulated mitochondrial pathway. Mol Pharmacol 84, 925-934 (2013).

53. K. S. Lee, J. Y. Liu, K. M. Wagner, S. Pakhomova, H. Dong, C. Morisseau, S. H. Fu, J. Yang, P. Wang, A. Ulu, C. A. Mate, L. V. Nguyen, S. H. Hwang, M. L. Edin, A. A. Mara, H. Wulff, M. E. Newcomer, D. C. Zeldin, B. D. Hammock, Optimized inhibitors of soluble epoxide hydrolase improve in vitro target residence time and in vivo efficacy. J Med Chem 57, 7016-7030 (2014).

54. D. Wan, J. Yang, C. B. McReynolds, B. Barnych, K. M. Wagner, C. Morisseau, S. H. Hwang, J. Sun, R. Blocher, B. D. Hammock, In vitro and in vivo Metabolism of a Potent Inhibitor of Soluble Epoxide Hydrolase, 1-(1-Propionylpiperidin-4-yl)-3-(4-(trifluoromethoxy)phenyl)urea. Front Pharmacol 10, 464 (2019).

55. A. Ghosh, T. Tyson, S. George, E. N. Hildebrandt, J. A. Steiner, Z. Madaj, E. Schulz, E. Machiela, W. G. McDonald, M. L. Escobar Galvis, J. H. Kordower, J. M. Van Raamsdonk, J. R. 
Colca, P. Brundin, Mitochondrial pyruvate carrier regulates autophagy, inflammation, and neurodegeneration in experimental models of Parkinson's disease. Sci Transl Med 8, 368ra174 (2016).

56. A. Ghosh, M. R. Langley, D. S. Harischandra, M. L. Neal, H. Jin, V. Anantharam, J. Joseph, T. Brenza, B. Narasimhan, A. Kanthasamy, B. Kalyanaraman, A. G. Kanthasamy, Mitoapocynin Treatment Protects Against Neuroinflammation and Dopaminergic Neurodegeneration in a Preclinical Animal Model of Parkinson's Disease. J Neuroimmune Pharmacol 11, 259-278 (2016).

57. A. Ghosh, A. Kanthasamy, J. Joseph, V. Anantharam, P. Srivastava, B. P. Dranka, B. Kalyanaraman, A. G. Kanthasamy, Anti-inflammatory and neuroprotective effects of an orally active apocynin derivative in pre-clinical models of Parkinson's disease. J Neuroinflammation $\mathbf{9}$, 241 (2012).

58. W. Guo, A. M. Allan, R. Zong, L. Zhang, E. B. Johnson, E. G. Schaller, A. C. Murthy, S. L. Goggin, A. J. Eisch, B. A. Oostra, D. L. Nelson, P. Jin, X. Zhao, Ablation of Fmrp in adult neural stem cells disrupts hippocampus-dependent learning. Nat Med 17, 559-565 (2011).

59. H. Lian, L. Yang, A. Cole, L. Sun, A. C. Chiang, S. W. Fowler, D. J. Shim, J. Rodriguez-Rivera, G. Taglialatela, J. L. Jankowsky, H. C. Lu, H. Zheng, NFkappaB-activated astroglial release of complement C3 compromises neuronal morphology and function associated with Alzheimer's disease. Neuron 85, 101-115 (2015).

60. J. Yang, K. Schmelzer, K. Georgi, B. D. Hammock, Quantitative profiling method for oxylipin metabolome by liquid chromatography electrospray ionization tandem mass spectrometry. Anal Chem 81, 8085-8093 (2009). 
Acknowledgements: We are grateful to V. Lee and J. Trojanowski (University of Pennsylvania) for providing postmortem brain tissues and T. Saito and T. Saido (RIKEN Brain Science Institute) for the APP $^{\text {NLGF }}$ knock-in mice. We thank Bianca Contreras and N. Aithmitti for expert technical assistance, B. Wang and E. Roy for help with organotypic culture and Nanostring experiments and members of the Zheng laboratory for insightful discussions. We acknowledge support from the Genomic and RNA Profiling Core and the Cytometry and Cell Sorting Core at Baylor College of Medicine for Nanostring and FACS analyses, respectively.

Funding: This project was supported by grants from the NIH (R01 NS093652, R01 AG020670, RF1 AG054111, R01 AG057509 and RF1 062257 to HZ) and NIH - NIEHS (RIVER Award R35 ES030443-01 and Superfund Research Program NIH - NIEHS P42 ES04699 to BDH)

Author Contributions: AG and HZ designed the overall study; AG performed all in vitro and in vivo assays and associated biochemical and immunohistochemical experiments and data analysis, with technical assistance from MC and NEP; MC and FC performed behavioral tests and LTP recording and associated data analysis, respectively; $\mathrm{SHH}$ and BDH provided TPPU and advised on the related experiments; DW performed mass spec analysis of oxylipins; AG and HZ wrote the manuscript and all authors read, provided input and approved the manuscript.

Competing interests: The authors declare no competing interests.

Data and materials availability: All data used for this study are included in the main manuscript or supplemental materials. 


\section{Figures and Figure Legends}

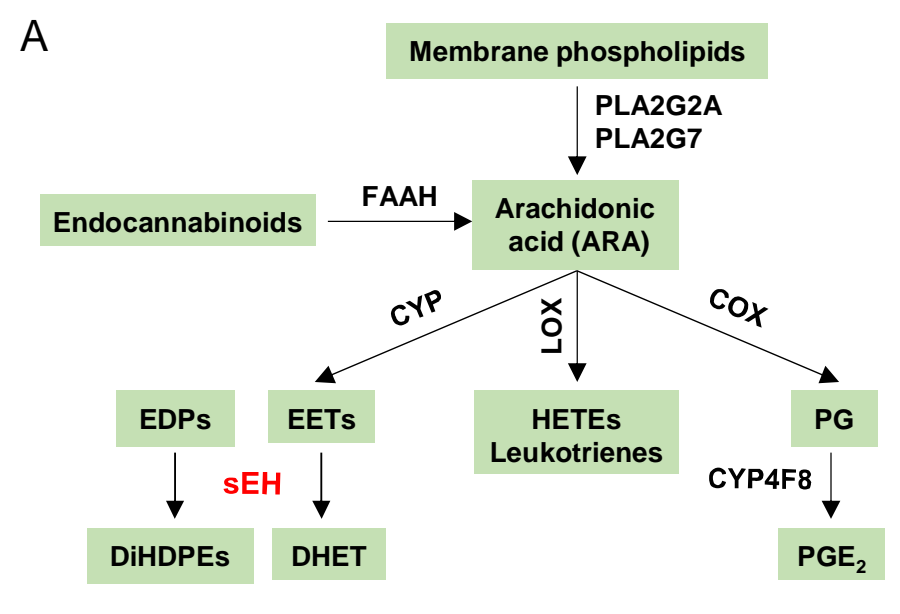

C

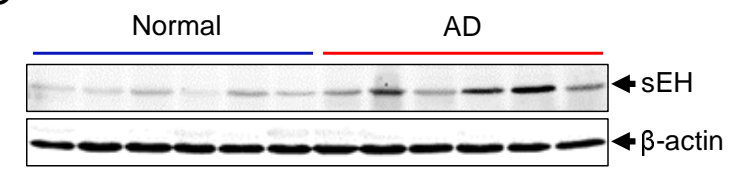

F

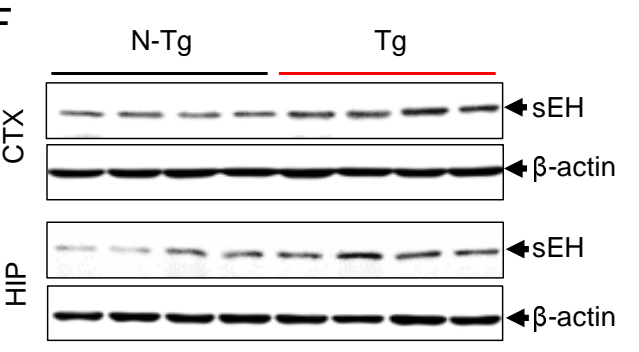

D
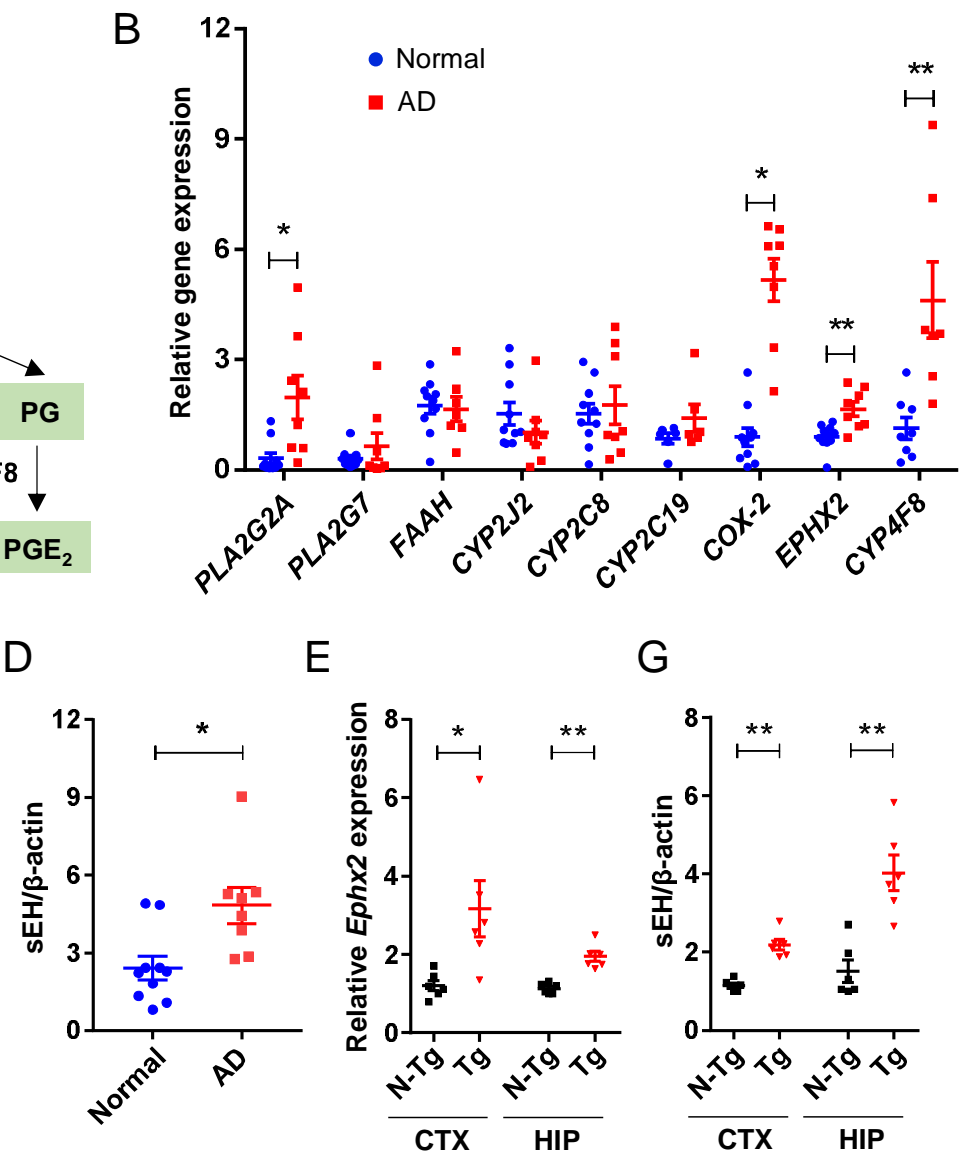

E

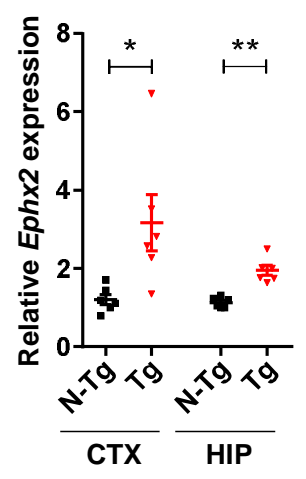

G

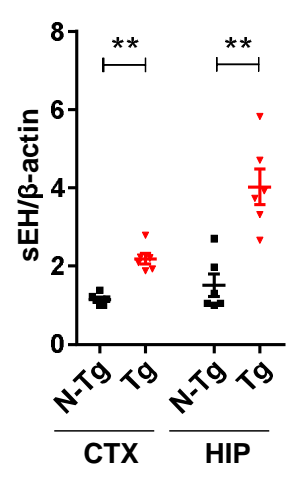

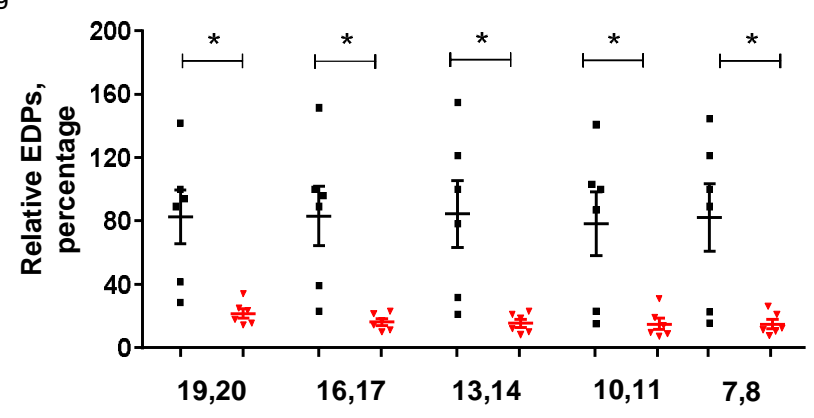


Fig. 1. sEH and arachidonic acid (ARA) metabolism dysregulation in AD. (A) Schematic diagram of ARA metabolism pathway. (B) qPCR analysis of mRNA expression of ARA pathway genes in the brains of AD patients $(n=8)$ and age-matched non-demented controls $(n=10)$. (C) Representative Western blot illustrating the expression of $\mathrm{sEH}$ in human brains. $\beta$-actin was used as a loading control. (D) Quantification of $\mathrm{sEH} / \beta$-actin ratio. (E) $\mathrm{qPCR}$ analysis of Ephx2 in cortex (CTX) and in hippocampus (HIP) of littermate non-transgenic (N-Tg) and 5xFAD transgenic (Tg) mice at 4.5 months of age. (F) Representative Western blot illustrating the levels in CTX and HIP of Tg mice and N-Tg controls. $\beta$-actin was used as a loading control. (G) Quantification of sEH/ $\beta$-actin ratio in CTX and in HIP. (H) Quantification of relevant EET and EDP regioisomers (displayed as percentage to a reference $\mathrm{N}-\mathrm{Tg}$ mouse) in $\mathrm{N}-\mathrm{Tg}$ and $\mathrm{Tg}$ mouse brains. The numbers at $\mathrm{x}$-axis denote carbon numbers where the double bonds were located in the corresponding polyunsaturated fatty acids. Data are means \pm SEM of eight to ten human brains per group (B-D) or six to eight mice per group $(\mathrm{E}-\mathrm{H})$. ${ }^{*} * \mathrm{P}<0.01,{ }^{*} \mathrm{P}<0.05$. Data were analyzed by unpaired Student's $t$-test. 
A

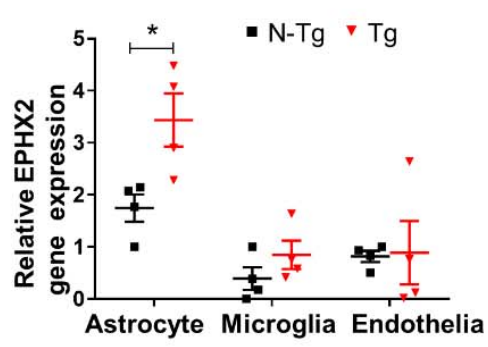

B
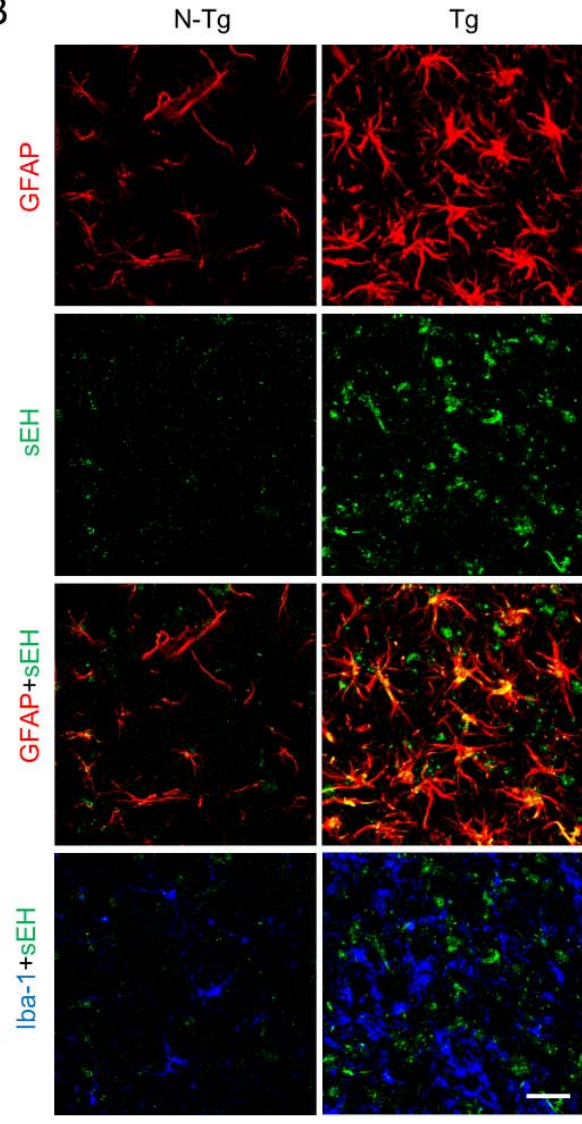

$\mathrm{H}$

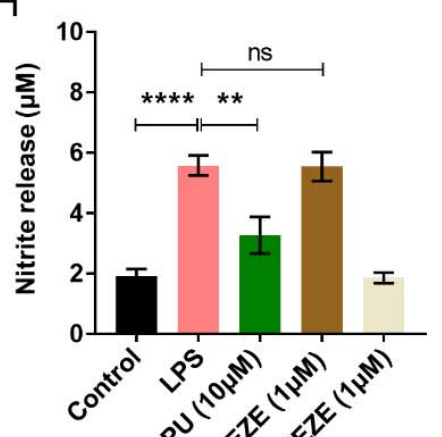

$\beta^{s^{x}} \beta^{\nu^{x}}$
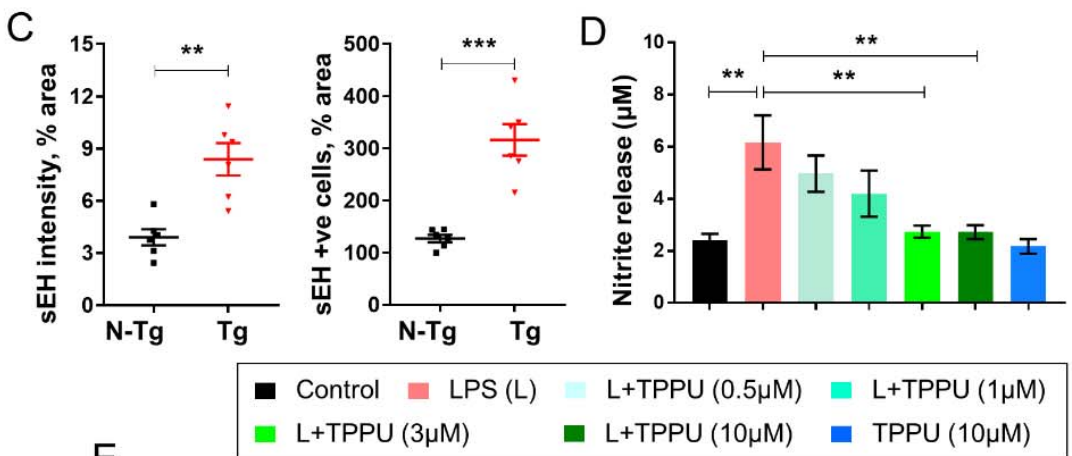

E
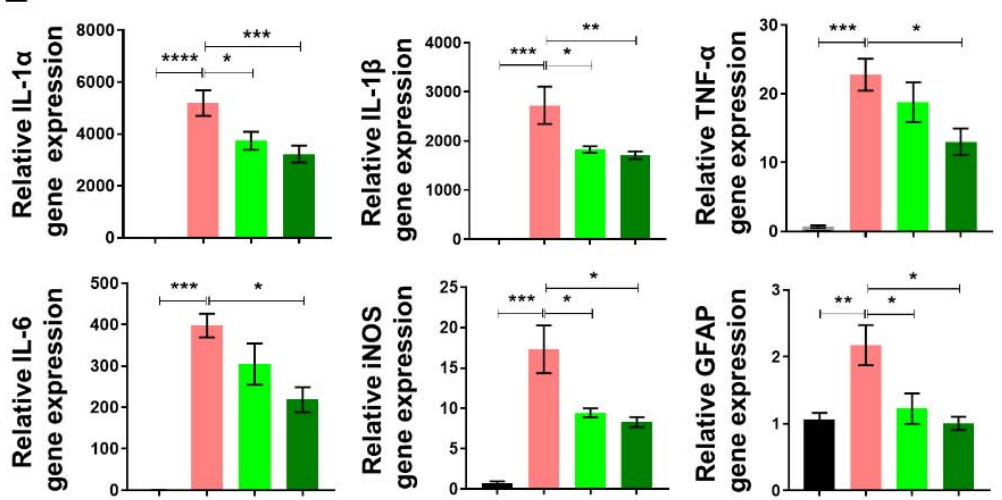

$\mathrm{F}$
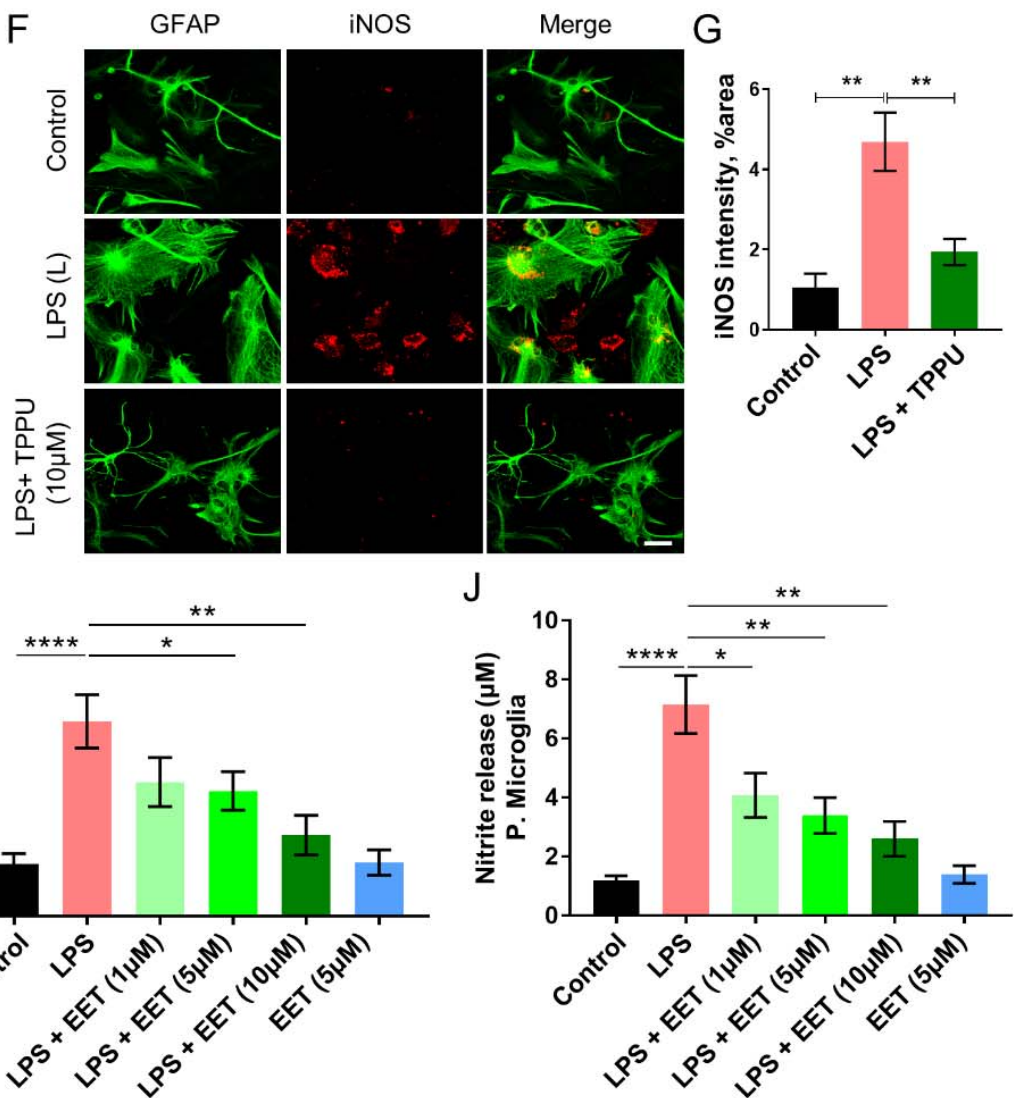
Fig. 2. Astrocytic sEH upregulation in Tg mice and by LPS treatment. (A) qPCR analysis of mRNA expression of Ephx2 in sorted astrocyte, microglia and endothelia of $\mathrm{N}-\mathrm{Tg}$ and $\mathrm{Tg}$ mice. (B) Immunostaining of GFAP (red), sEH (green) and Iba-1 (blue) in the hippocampus of Tg mice at 4.5 months of age. Scale bar $50 \mu \mathrm{m}$. (C) Quantification of sEH intensity (left) and sEH+ve cells (right) from (B). (D-G) Analysis of conditioned media or cell lysates of primary astrocyte cultures pretreated with different doses of TPPU (ranging from $0.5 \mu \mathrm{M}$ to $10 \mu \mathrm{M}$ ) for 30 minutes followed by LPS treatment (100 ng/ml) for $24 \mathrm{~h}$. (D) Nitrite measurement from cultured media by Griess assay. (E) qPCR analysis of mRNA expression of $I l-1 \alpha, I l-1 \beta, T n f-\alpha, I l-6$, iNOS and Gfap. (F) Immunocytochemistry of GFAP (green) and iNOS (red) in primary astrocytes. Scale bar, $100 \mu \mathrm{m}$. (G) Quantification of iNOS intensity in primary astrocytes. $(\mathrm{H})$ Nitrite measurement of conditioned media from primary astrocytes pretreated with TPPU $(10 \mu \mathrm{M})$ or pan-EET receptor antagonist 14,15-EEZE $(1 \mu \mathrm{M})$ for 30 minutes followed by LPS treatment $(100 \mathrm{ng} / \mathrm{ml})$ for 24 hours. ( $\mathrm{i}$ and $\mathrm{j}$ ) Nitrite levels in conditioned media of primary astrocytes (I) or primary microglia (J) pretreated with different doses of 11,12-EET (ranging from $1 \mu \mathrm{M}$ to $10 \mu \mathrm{M})$ for 30 minutes followed by LPS treatment $(100 \mathrm{ng} / \mathrm{ml})$ for $24 \mathrm{~h}$. Data are means \pm SEM of either four to six mice per group (A-C) or three independent experiments $(\mathrm{n}=3, \mathrm{D}-\mathrm{J}) . * * * * \mathrm{P}<0.0001$, $* * * \mathrm{P}<0.001, * * \mathrm{P}<0.01, * \mathrm{P}<0.05, \mathrm{~ns}=$ not significant. Data were analyzed either by unpaired Student's $t$-test (A-D) or by one-way ANOVA with Tukey's multiple comparison test (D-J). 


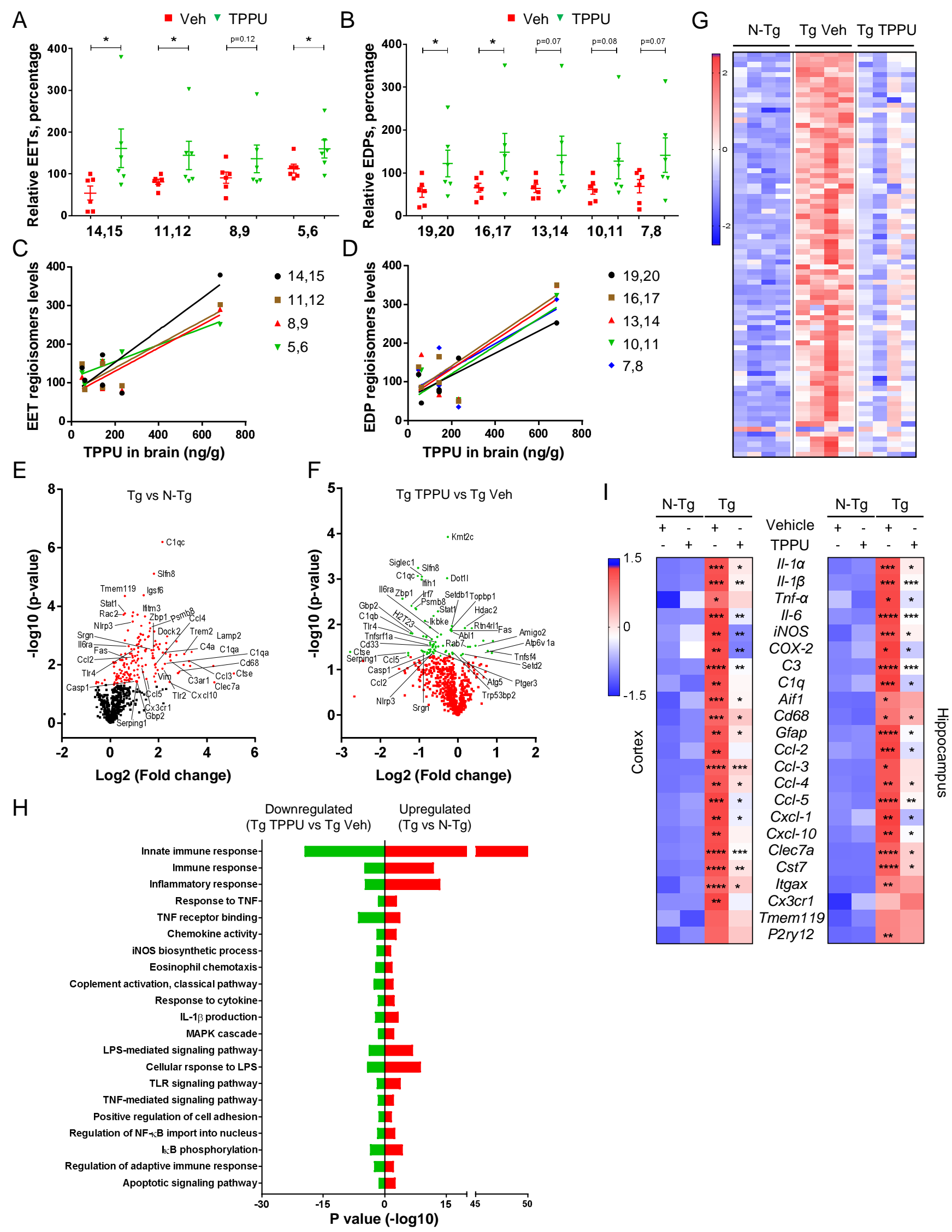




\section{Fig. 3. TPPU engages its target and attenuates inflammatory gene expression in Tg mice. (A, B)}

Quantification of relevant EETs (A) and EDPs (B) (displayed as percentage to a reference Tg Veh mouse) from vehicle (Veh) and TPPU-treated mouse brains. (C) Correlation of brain TPPU with different EET regioisomers ( $\mathrm{r}=0.874$, $\mathrm{p}<0.0238$ for 14,15-EET; $\mathrm{r}=0.866, \mathrm{p}<0.026$ for 11,12-EET; $\mathrm{r}=0.02$ for 8,9-EET; $\mathrm{r}=0.947, \mathrm{p}<0.004$ for 5,6-EET). (D) Correlation of brain TPPU with different EDP regioisomers $(\mathrm{r}=0.899, \mathrm{p}<0.015$ for $19,20-\mathrm{EDP} ; \mathrm{r}=0.84, \mathrm{p}<0.037$ for $16,17-\mathrm{EDP} ; \mathrm{r}=0.796, \mathrm{p}<0.581$ for 13,14-EDP; r=0.84, p<0.036 for 10,11-EDP; $r=0.762$, p<0.079 for 7,8-EDP). (E, F) Volcano plot of Nanostring neuroinflammation gene expression profiling showing differences in gene expression in the hippocampus stratified by (E) Tg vs N-Tg and (F) Tg TPPU vs Tg Veh. For each plot, significance is plotted against fold-change (log2 values). Red dots and green dots denote genes with adjusted significance of $\mathrm{p}<0.05$. (G) Heat map of relative expression of inflammatory pathway genes in $\mathrm{N}-\mathrm{Tg}$, $\mathrm{Tg}$ Veh and Tg TPPU mice. (H) Gene ontology (GO) and pathway analysis of differentially expressed genes (DEGs). Significant GO terms (biological processes) associated with identified DEGs. The vertical axis represents the GO category, and the horizontal axis represents the P-value $(-\log 10)$ of the significant GO terms. Red bars represent the significantly upregulated inflammatory pathways in $\mathrm{Tg}$ mice vs N-Tg mice and green bars represent significantly downregulated inflammatory pathways in $\mathrm{Tg}$ TPPU mice vs Tg Veh mice. (I) Heat map showing qPCR analysis of mRNA expression in cortex (left) and in hippocampus (right). The asterisks in Tg: Vehicle (+) TPPU (-) column represent significant change vs N-Tg: Vehicle (+) TPPU (-). The asterisks in Tg: Vehicle (-) TPPU (+) represent significant change vs Tg: Vehicle (+) TPPU (-). Data are means \pm SEM of either six to eight mice (A-D) or four mice (E-H) per group. Data were analyzed by Student's $t$-test or by two-way ANOVA with Bonferroni's multiple comparison test. For C and D, correlation coefficients (r) were computed using Pearson correlations and each dot represents individual mouse and linear regression line (solid). 
A

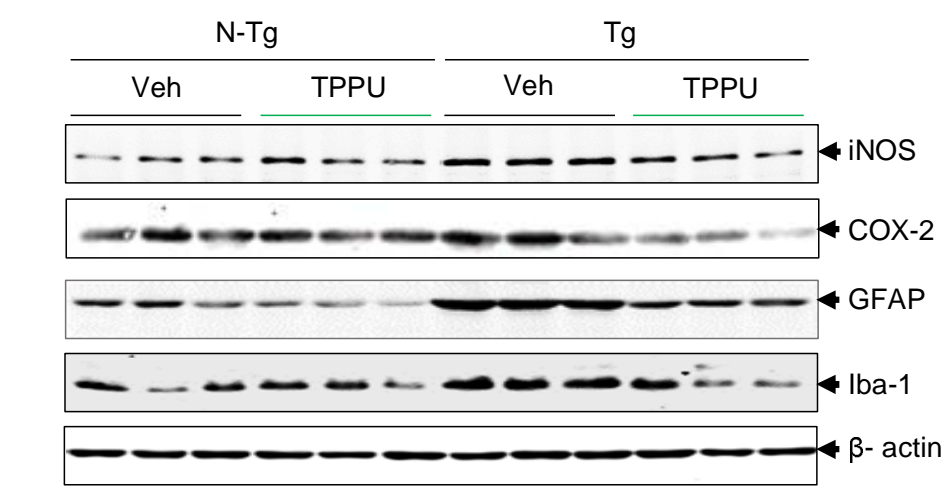

C
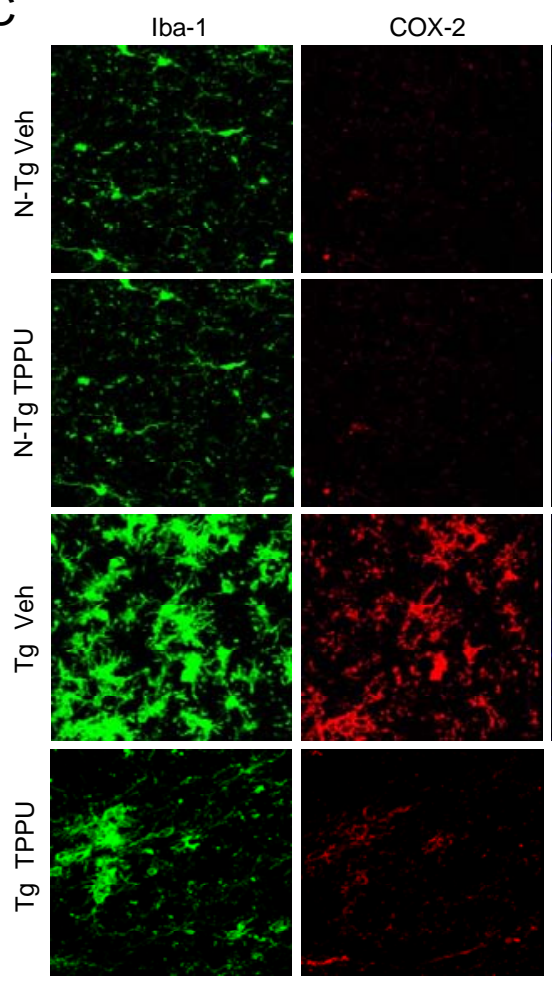

iNOS
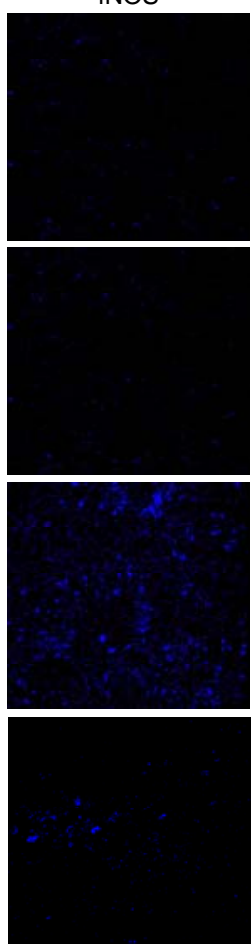
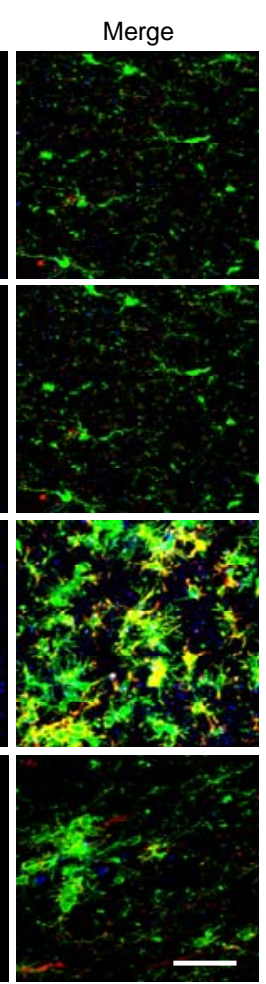

B
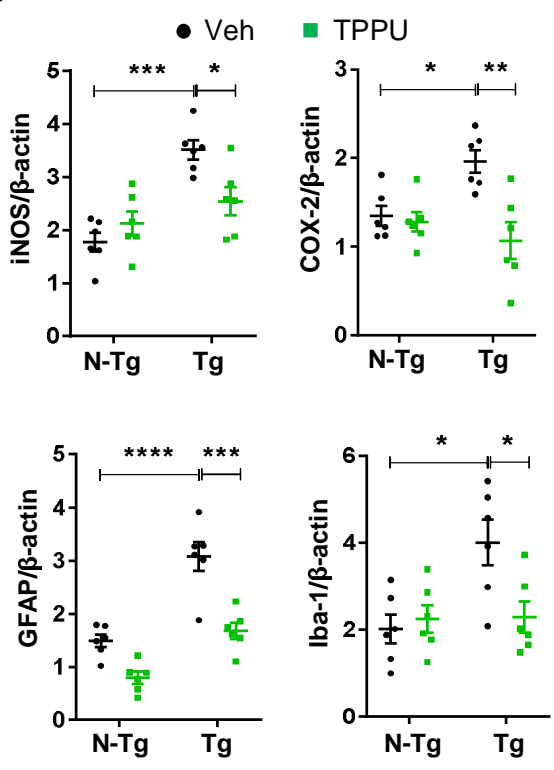

$\mathrm{D}$
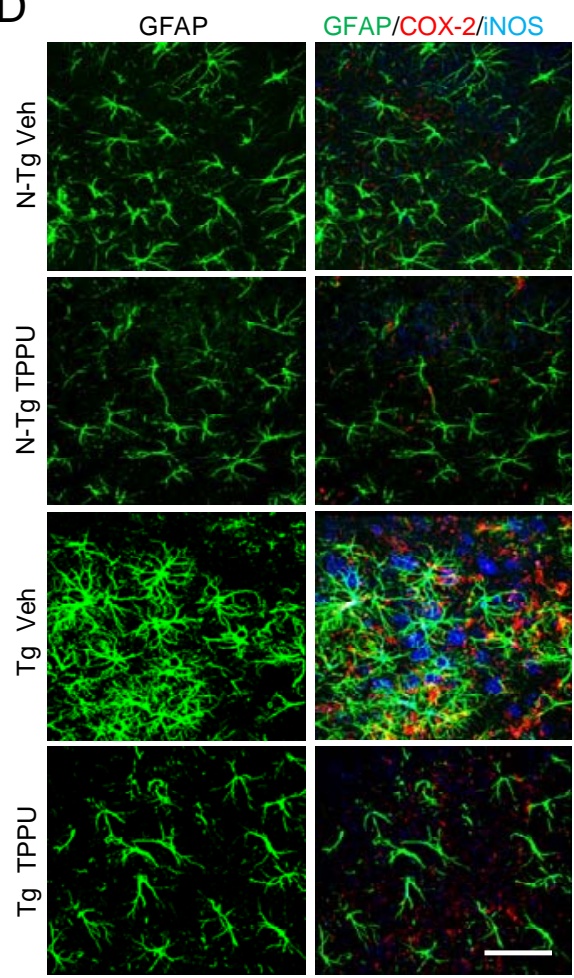

$\mathrm{E}$

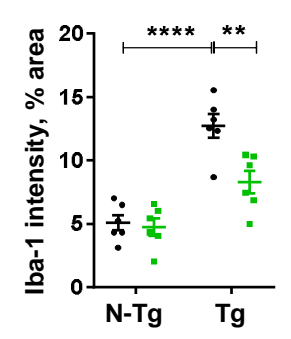

- Veh — TPPU

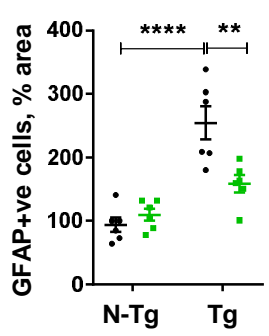

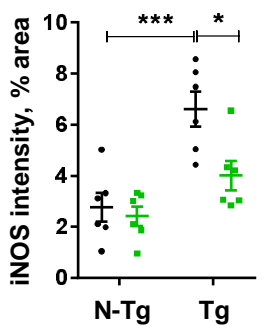

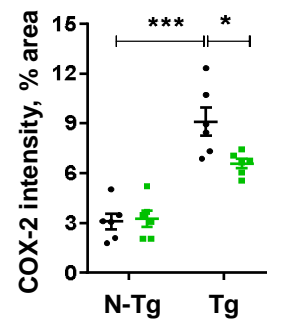


Fig. 4. TPPU reduces neuroinflammatory markers and gliosis in $\mathbf{T g}$ mice. (A) Representative Western blot of iNOS, COX-2, GFAP and Iba-1 in hippocampal samples of N-Tg and Tg mice treated with vehicle (Veh) or TPPU starting at 2 months for 2.5 months. $\beta$-actin was used as a control. (B) Quantification of (A). (C) Triple immunofluorescence staining of Iba-1 (green), COX-2 (red) and iNOS (blue) in hippocampus of above mice. Scale bar, $50 \mu \mathrm{m}$. (D) Immunofluorescence staining of GFAP (left) and merged panel of GFAP (green), COX-2 (red) and iNOS (blue) (right). Scale bar, $50 \mu \mathrm{m}$. (E) Quantification of Iba-1, iNOS and COX-2 intensities and Iba-1+ve and GFAP+ve cells in the hippocampus. Data are means \pm SEM of six to eight mice per group. $* * * * \mathrm{P}<0.0001, * * * \mathrm{P}<0.001, * * \mathrm{P}$ $<0.01, * \mathrm{P}<0.05$. Data were analyzed by two-way ANOVA with Bonferroni's multiple comparison test. 


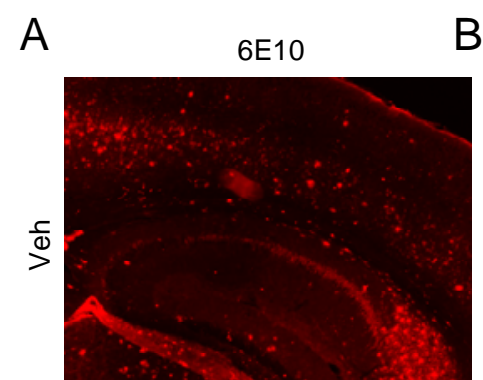

B
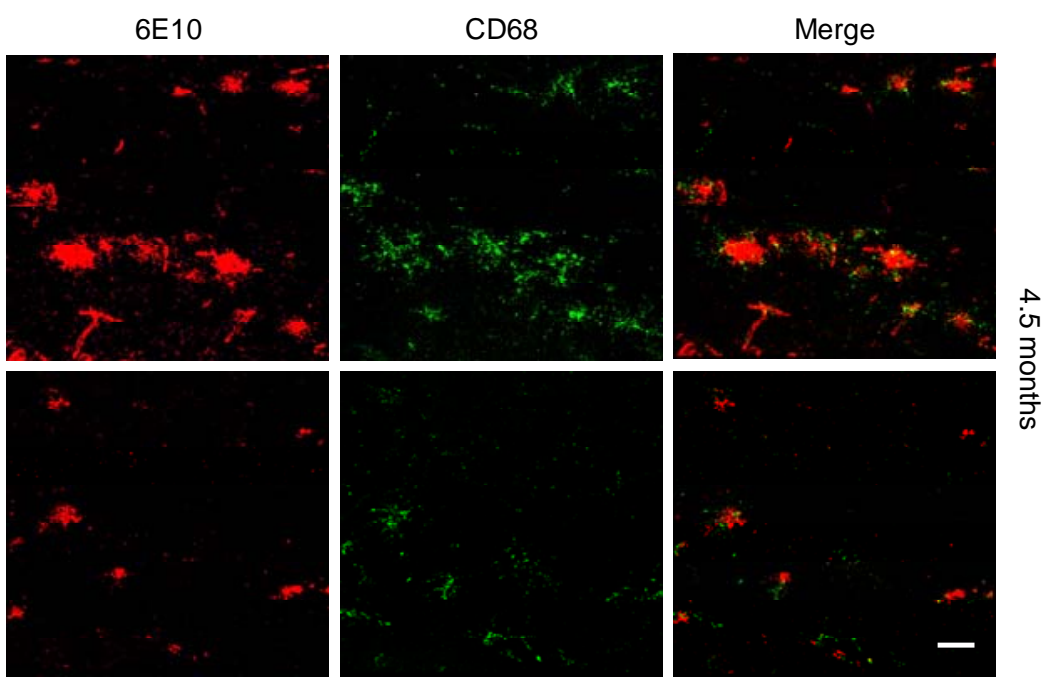

C
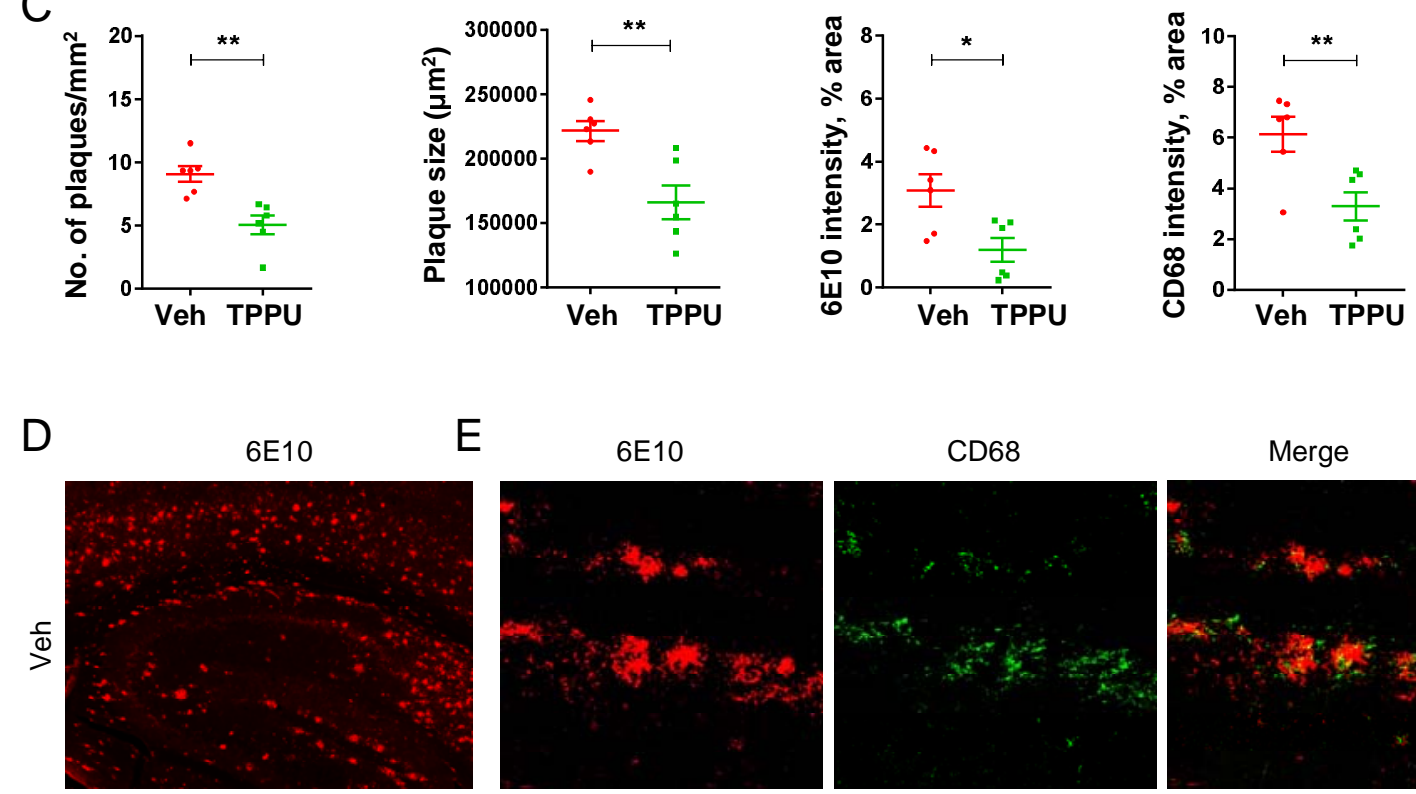

E
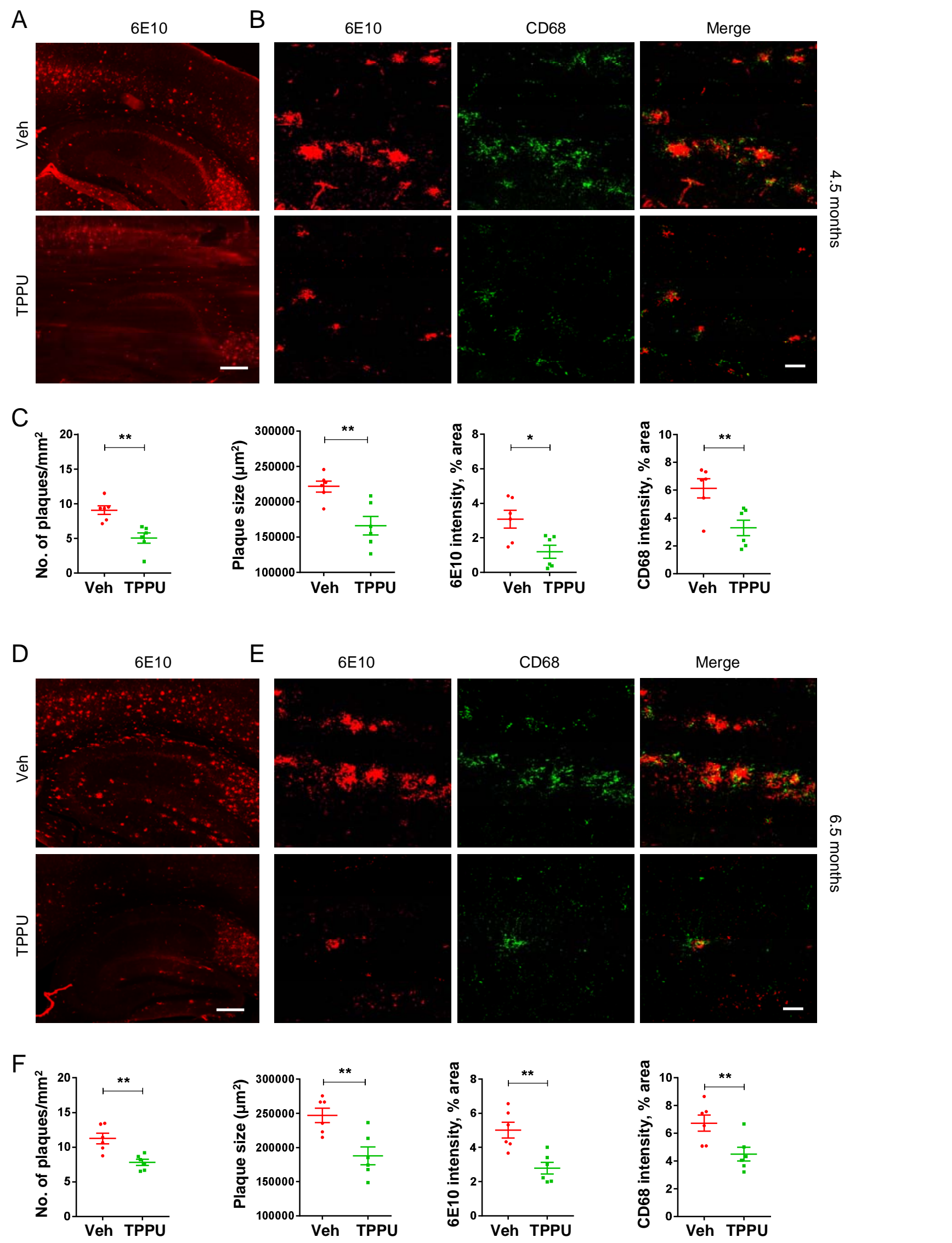
Fig. 5. TPPU reduces A $\boldsymbol{\beta}$ burden in Tg mice. (A) Immunohistochemistry of $6 \mathrm{E} 10$ (lower magnification) in Tg mice treated with vehicle (Veh) or TPPU starting at 2 months for 2.5 months. Scale bar, $400 \mu \mathrm{m}$. (B) Higher magnification of hippocampus of Tg Veh or TPPU mice with double immunofluorescence staining of 6E10 (red) and CD68 (green). Scale bar, $200 \mu \mathrm{m}$. (C) Quantification of 6E10+ve plaque number, size and 6E10 and CD68 intensities in the hippocampus. (D-F) The same analysis and data presentation as (A-C) except mice treated with Veh or TPPU starting at 2 months for a duration of 4.5 months were analyzed. Data are means \pm SEM of six to eight mice per group. $* * \mathrm{P}<$ $0.01, * \mathrm{P}<0.05$. Data were analyzed by Student's $t$-test. 
A
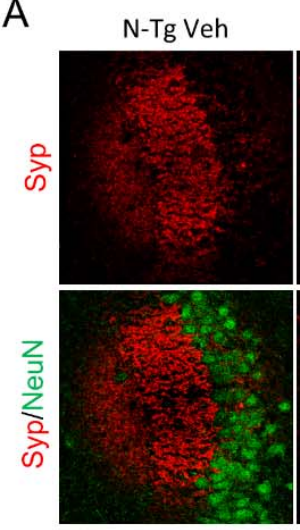

C

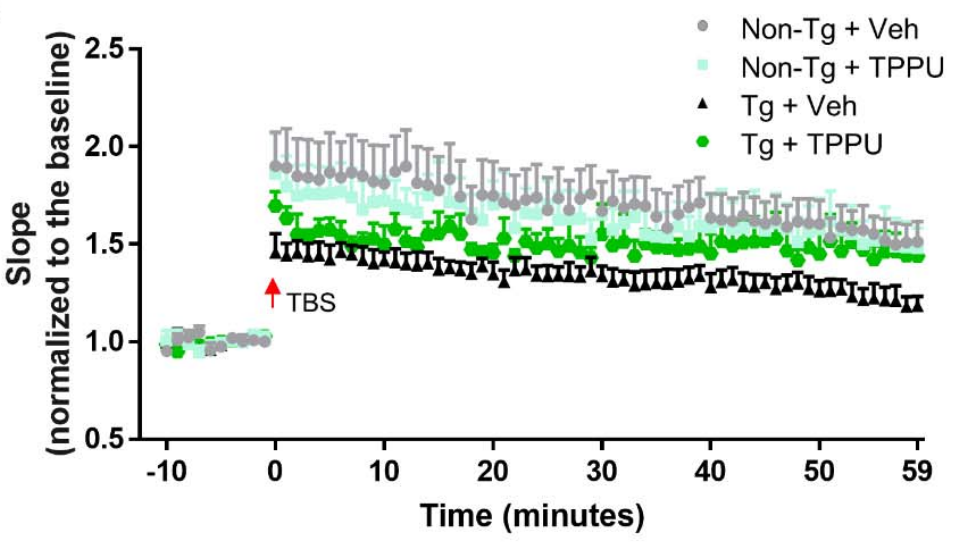

B
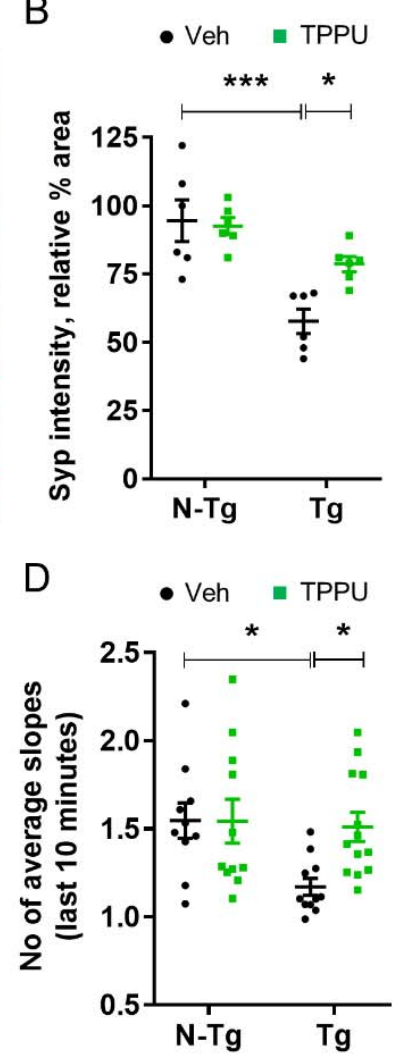

E

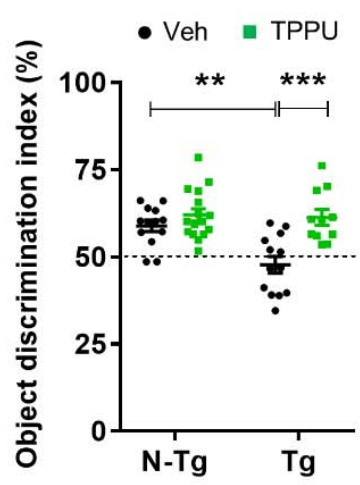

F
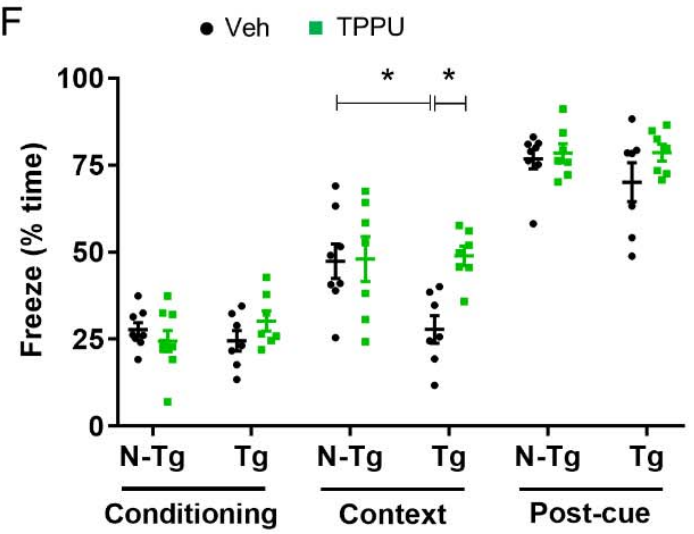
Fig. 6. TPPU ameliorates synaptic deficits and cognitive function in Tg mice. (A) Representative images of synaptophysin (Syp) and NeuN co-immunostaining of area CA3 of hippocampus of N-Tg and Tg mice treated with vehicle (Veh) or TPPU starting at 2 months for 4.5 months. Scale bar, $100 \mu \mathrm{m}$. (B) Quantification of Syp intensity. (C) Slope of field excitatory postsynaptic potential (fEPSP) in response to theta burst stimulation delivered to the Schaffer collateral pathway from the above mice. Calibration: $2 \mathrm{mV}, 5 \mathrm{~ms} . \mathrm{n}=10-13$ sections from 5-6 animals per group/genotype. (D) No of average fEPSP slopes (last 10 minutes). (E) Object Discrimination Index of Veh- or TPPU-treated N-Tg or Tg mice in a novel object recognition test. The dotted line represents the $50 \%$ chance of random object exploration. (F) Percent of freezing in Veh- or TPPU-treated N-Tg or Tg mice in the Conditioning (left), Contextual (center) and cued (right) fear conditioning tests. Mice with 2.5 months Veh or TPPU treatment were subjected to behavior analysis. Values are expressed as mean \pm SEM of either six to nine mice per group (A-D and F) or twelve to sixteen mice per group (E). Data were analyzed by two-way ANOVA with Bonferroni's multiple comparison test. $* * * \mathrm{P}<0.001, * * \mathrm{P}<0.01, * \mathrm{P}<0.05$. 